Please do not remove this page

RMIT

UNIVERSITY

\title{
Automated modal parameter-based anomaly detection under varying wind excitation
}

Neu, Eugen; Janser, Frank; Afaghi Khatibi, Akbar; Orifici, Adrian

https://researchrepository.rmit.edu.au/esploro/outputs/9921860536801341/filesAndLinks?institution=61RMIT_INST\&index=null

Neu, E., Janser, F., Afaghi Khatibi, A., \& Orifici, A. (2016). Automated modal parameter-based anomaly detection under varying wind excitation. Structural Health Monitoring, 15(6), 730-749.

https://doi.org/10.1177/1475921716665803

Document Version: Accepted Manuscript

Published Version: https://doi.org/10.1177/1475921716665803

Repository homepage: https://researchrepository.rmit.edu.au

(c) The Author(s) 2016

Downloaded On 2023/04/26 20:11:23 +1000

Please do not remove this page 
Thank you for downloading this document from the RMIT Research Repository.

The RMIT Research Repository is an open access database showcasing the research outputs of RMIT University researchers.

RMIT Research Repository:http://researchbank.rmit.edu.au/

\section{Citation:}

Neu, E, Janser, F, Afaghi Khatibi, A and Orifici, A 2016, 'Automated modal parameter-based anomaly detection under varying wind excitation', Structural Health Monitoring, vol. 15, no. 6, pp. 730-749.

See this record in the RMIT Research Repository at:

https://researchbank.rmit.edu.au/view/rmit:39910

Version: Accepted Manuscript

Copyright Statement: (c) The Author(s) 2016

Link to Published Version:

http://dx.doi.org/10.1177/1475921716665803 


\title{
Automated Modal Parameter-based Anomaly Detection under varying Wind Excitation
}

\author{
Eugen Neu (neu@fh-aachen.de) $)^{1,2}$, Frank Janser ${ }^{1}$, Akbar A. Khatibi ${ }^{2}$ and Adrian C. Orifici ${ }^{2}$ \\ ${ }^{1} \mathrm{FH}$ Aachen UAS, Hohenstaufenallee 6, Aachen, Germany \\ ${ }^{2}$ RMIT University, GPO Box 2476 Melbourne, Victoria Australia, 3001
}

\begin{abstract}
Wind-induced operational variability is one of the major challenges for structural health monitoring of slender engineering structures like aircraft wings or wind turbine blades. Damage sensitive features often show an even bigger sensitivity to operational variability. In this study a composite cantilever was subjected to multiple mass configurations, velocities and angles of attack in a controlled wind tunnel environment. A small-scale impact damage was introduced to the specimen and the structural response measurements were repeated. The proposed damage detection methodology is based on automated operational modal analysis. A novel baseline preparation procedure is described that reduces the amount of user interaction to the provision of a single consistency threshold. The procedure starts with an indeterminate number of operational modal analysis identifications from a large number of datasets and returns a complete baseline matrix of natural frequencies and damping ratios that is suitable for subsequent anomaly detection. Mahalanobis distance-based anomaly detection is then applied to successfully detect the damage under varying severities of operational variability and with various degrees of knowledge about the present operational conditions. The damage detection capabilities of the proposed methodology were found to be excellent under varying velocities and angles of attack. Damage detection was less successful under joint mass and wind variability but could be significantly improved through the provision of the currently encountered operational conditions.
\end{abstract}

Keywords - structural health monitoring; baseline; training set; wind; environmental and operational variability; operational modal analysis

\section{Introduction}

Modal parameters have several unique properties that make them excellent damage-sensitive feature candidates for Structural Health Monitoring (SHM). They are by far the single best investigated and understood dynamic system property. Their sensitivity to damage was proven in a myriad of analytical, numerical and experimental studies $[1,2,3,4]$. They can be used to tune numerical models from experimental data and in the opposite direction to test experimentally-trained SHM systems with numerically generated damage scenarios. The major disadvantage of using modal parameters as damage-sensitive features is the non-trivial and sometimes unreliable automated modal parameter identification process. Until recently no robust automatic modal parameter extraction techniques existed and modal parameters were manually selected by experienced users. Over the last decade the automated extraction of modal properties from ambiently excited structures has received significantly more attention and a number of Automated Operational Modal Analysis (AOMA) methodologies have been proposed [5, 6, 7, 8]. However, only a small number of studies were published were AOMA was integrated into a fully functional SHM system and tested with experimental data $[9,10,11,12]$. All hitherto proposed full SHM methodologies that are based on AOMA rely on manual user interaction and on manually tuned parameters. The setup process heavily relies on user experience and expert knowledge. Furthermore, consideration of Operational and Environmental Variability (OEV) remains a challenging task, especially when neither explicit measurements of the OEV nor representative samples of the outlier class are available. 
The success of a SHM system strongly depends, among other things, on the careful preparation of the training data. General AOMA algorithms return an indeterminate number of modes from each dataset. These have to be sorted into coherent groups and filtered for consistency. Pre-processing for AOMA-based SHM usually involves iterative data processing and manual user interaction. The exact procedure is rarely described since it involves manual parameter tuning, repetitive visualisation and re-partitioning and sometimes even the manual assignment of observations into clusters based on expert judgement. The handling of closely spaced natural frequencies is especially challenging. Deraemaeker et al. [13] investigated a vibration-based SHM methodology using a numerical bridge model. They manually selected the initial set of baseline modes from a single dataset using manual Operational Modal Analysis (OMA) and determined appertaining modes from other datasets through a frequency-based modal tracking procedure. Devriendt et al. [7] investigated a parked wind turbine and manually selected the modes of interest from a single reference dataset as well. They introduced a two-stage tracking procedure based on frequency distances and Modal Assurance Criterion (MAC). The procedure limits the maximum MAC and frequency shifts between the reference modes and the modes obtained through tracking to 0.8 and $5 \%$ respectively. Schwochow and Jelicic [14] proposed a pole-weighted MAC for tracking in the context of in-flight flutter detection. Again, modes were only tracked within an arbitrary chosen but not further specified region around the manually chosen reference modes. Magalhães et al. [11] showed results of a 2 year arch bridge monitoring campaign but did not describe the baseline preparation or tracking procedure used. Reynders et al. [15] used AOMA to extract four natural frequencies that were used as features in a damage detection study on a three-span concrete bridge. Again, no details were given on training set preparation. All the listed studies have in common that substantial manual effort is required or that a number of case-dependent parameters have to be selected during the setup procedure before the AOMA identifications are transformed into a form that is suitable for statistical data modelling.

$\mathrm{OEV}$ is one of the major obstacles to the comprehensive introduction of continuous vibration-based SHM. Multiple approaches have been proposed to account for OEV. The most straightforward approach is the direct measurement of the dependent variables (e.g. natural frequencies, damping ratios, mode shapes) and independent operational and environmental variables (e.g. temperature, wind velocity, wind direction). A (static) regression model can then be used to remove the OEV, effectively creating new, OEV-normalised features [16]. Regression models with temporal terms can be used to capture dynamic interactions. Peeters and de Roeck [9] used an Autoregressive Exogenous (ARX) model to capture the dynamics between temperature and natural frequency shifts. These or similar approaches can be used to model and monitor operation points that were not present in the original training set, as long as they can be represented by the regression model. However, direct and thorough measurement of all relevant OEV is seldom possible. Another approach is to get sufficient samples under all encounterable operational and environmental conditions. In the context of SHM a number of dimensionality reduction or decomposition techniques like Factor Analysis (FA), Principal Component Analysis (PCA) or Singular Value Decomposition (SVD) were proposed to identify the OEV from the baseline and distinguish these changes from damage-induced changes $[13,17]$. The problem can also be stated as a data domain description or oneclass classification problem [18]. Partial measurements of the OEV can be incorporated into the domain description approach by appending the Operational and Environmental Conditions (OEC) measurements to the feature vector. The vast majority of SHM studies under OEV concentrate on temperature variability $[11,15,9]$. Devriendt et al. [7] also investigated the influence of tidal levels. In laboratory studies OEV is often simulated through mass and/or stiffness modifications [16, 19]. Damage detection under wind-induced operational variability was not thoroughly investigated yet. Specifically, limited publications have considered the effect of wind velocity, and no publications to date have considered the effect of Angle Of Attack (AOA), which are both critical to understand for aircraft and wind turbine blade applications. Peeters and de Roeck [9] found no relationship between the four extracted natural frequencies of a concrete bridge and the measured wind characteristics. However, it is well known that wind characteristics have a substantial influence on modal properties, especially if the structure under consideration is a slender cantilever [20, 21, 22, 23].

This study introduces two novel contributions. First, a fully automated baseline preparation methodology is described that starts with individual AOMA identifications from a large number of datasets $M$. 
The number of modes identified from each of these datasets is indeterminate and they are not grouped in any way. The goal of the automated baseline generation procedure is to single out consistently identified modes and order them into cohesive sets. The end product of the procedure is the $N \times p$ matrix $\mathbf{X}$, where each row of $\mathbf{X}$ represents one measurement of the feature vector $x_{i}^{T} . p$ is the number of features extracted from the selected modal properties. The only user-defined parameter of the procedure is the desired consistency ratio $N / M$, which can be directly related to the anticipated false alarm rate. The procedure does not include any arbitrarily chosen thresholds. Instead, clustering and statistical modelling is used to derive any necessary thresholds from the actual data. This, in principle, allows the procedure to automatically adapt to different sensor numbers and types, OEV intensities and inter-frequency distances. Second, the aforementioned baseline preparation method is integrated into a full SHM system to detect an impact damage on a composite cantilever. The cantilever was subjected to varying wind velocities and angles of attack in a wind tunnel. Furthermore, the mass of the specimen was slightly modified in two steps to introduce a third operational variability. The specimen was then subjected to an impact damage and all measurements were repeated. The damage-induced modal parameter changes are significantly smaller than the variability introduced by the OEV. Damage detection is investigated under different severities of operational variability as well as with and without explicit measurements of the OEC.

\section{Methodology}

\subsection{Experimental setup and data}

To assess the performance of the proposed SHM methodology under varying wind excitation experimental data from a wind tunnel study are used. The experimental setup is shown in figure 1. The investigation was conducted in a closed-loop wind tunnel with an open test section. The investigated specimen was a glass fiber-reinforced polymer plate $(500 \mathrm{~mm} \times 90 \mathrm{~mm} \times 4 \mathrm{~mm})$, which was subjected to different flow conditions. The structural response of the specimen was measured using three sensor types: Fiber Bragg Grating Sensorss (FBGSs), a unidirectional piezoelectric (PZT) accelerometer and a piezoelectric strain sensor. Only measurements from the two piezoelectric sensors are used in this study. These were recorded at a sampling rate of $1600 \mathrm{~Hz}$. The dynamic properties of the inflowing wind were measured using a hot-wire anemometer. Three angles of attack $\alpha_{1 \ldots 3}=0^{\circ}, 1^{\circ}, 2^{\circ}$ and three inflow velocities $\mathrm{v}_{1 \ldots 3}=$ $70 \mathrm{~km} / \mathrm{h}, 100 \mathrm{~km} / \mathrm{h}, 130 \mathrm{~km} / \mathrm{h}$ were investigated. The dynamic pressure is doubled at every velocity step. Three mass configurations were investigated: The $390 \mathrm{~g}$ specimen without additional mass $\left(\mathrm{m}_{0}\right)$, an additional $16 \mathrm{~g}$ mass at the upper attachment point $\left(\mathrm{m}_{1}\right)$, a second additional $16 \mathrm{~g}$ mass at the lower attachment point $\left(\mathrm{m}_{2}\right)$. The two mass attachment points are shown in figure 1. All measurements were repeated after damage was introduced to the specimen. In total 54 individual operation points were measured. Measurements were conducted for approximately 40 minutes at every operation point and split into 64 equally sized datasets per operation point. Further information about the experimental setup were published in [22].

The result of the impact damage is shown in figure 2. The damage was created with a drop test rig. A damage scenario was chosen through pre-testing that created a modal parameter shift in the same range as the investigated OEV. The dimensions of the final damage are approximately $20 \mathrm{~mm} \times 20 \mathrm{~mm}$. The $20 \mathrm{~J}$ impact resulted in a palpable dent on the impact side and clearly visible fiber breakage on the opposite side. The damage was introduced at the symmetry plane of the specimen, placed at the height of the piezoelectric strain sensor. The former location of the piezoelectric sensor is indicated with red color in figure 2 .

\subsection{Definitions}

The relative difference between scalar (real or complex) values $X_{i}$ and $X_{j}$ is calculated using the formulation in equation (1) throughout this text. 


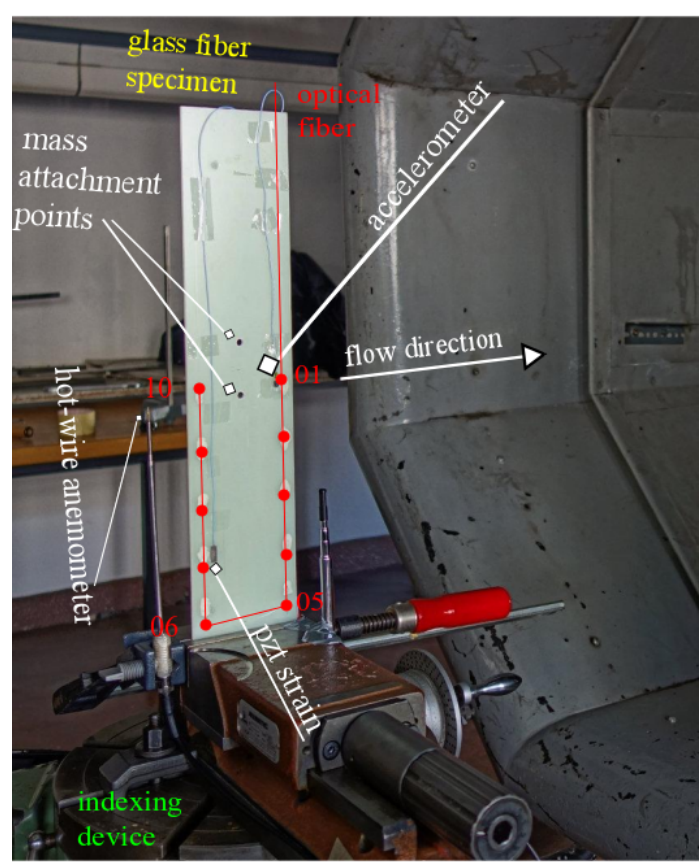

Figure 1: Wind tunnel setup.

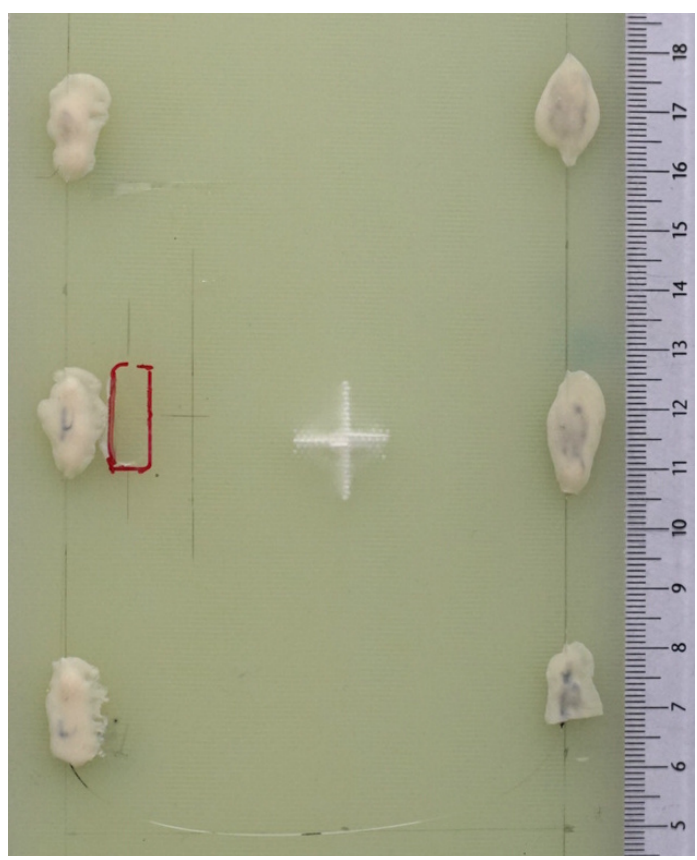

Figure 2: Impact damage (white cross).

$$
d X_{i, j}=\frac{\left|X_{i}-X_{j}\right|}{\max \left(\left|X_{i}\right|,\left|X_{j}\right|\right)}
$$

Equation (1) is used to measure the relative natural frequency distance $d f_{u}$, the relative damping distance $d \xi$, the relative pole distance $d \lambda$ and the relative mean phase deviation $d M P D$. The Modal Assurance Criterion (MAC), which defines a relative correlation between two modes, is defined according to Eq. (2). 


$$
M A C_{i, j}=\frac{\left|\phi_{i}^{T} \cdot \phi_{j}^{*}\right|^{2}}{\left(\phi_{i}^{T} \cdot \phi_{i}^{*}\right)\left(\phi_{j}^{T} \cdot \phi_{j}^{*}\right)}
$$

where $\phi_{i}$ and $\phi_{j}$ are mode shapes, which can be either real or complex. The Mean Phase Deviation (MPD) is a measure of mode shape complexity. It describes the mean phase angle deviation of the individual mode shape components from a straight line in the complex plane. It is calculated using a total least squares fit of the mode shape in the complex plane (Eq. (3)) and a weighted sum of phase angle deviations (Eq. (4)) [6].

$$
\begin{aligned}
\mathbf{U S V}^{T} & =\left[\operatorname{Re}\left(\phi_{i}\right) \operatorname{Im}\left(\phi_{i}\right)\right] \\
M P D_{i} & =\frac{\sum_{n=1}^{N_{\phi}} w_{n} \arccos \left|\frac{\operatorname{Re}\left(\phi_{j n}\right) V_{22}-\operatorname{Im}\left(\phi_{i n}\right) V_{12}}{\sqrt{V_{12}^{2}+V_{22}^{2}}\left|\phi_{i n}\right|}\right|}{\sum_{n=1}^{N_{\phi}} w_{n}}
\end{aligned}
$$

where $V_{12}$ and $V_{22}$ are the individual components of the right singular matrix of the singular value decomposition $\mathbf{U S V}^{T} . N_{\phi}$ is the number of mode shape components, $w_{n}$ are weighting factors that are chosen as $\left|\phi_{i n}\right|$ in this work.

\subsection{Automatic preparation of the baseline dataset}

The automated baseline preparation procedure described in this section is one of the major contributions of this study. The goal is to significantly reduce user interaction and to automatically derive necessary parameters from the statistical properties of the actual data.

\subsubsection{Automated operational modal analysis input data}

The procedure starts with AOMA identifications from a large number of baseline datasets $M$, where $m=1,2, \ldots, M$. Each AOMA baseline dataset consists of $K_{m}$ poles $\lambda_{1} \ldots \lambda_{K_{m}}$ and $K_{m}$ unscaled mode shapes $\phi_{1} \ldots \phi_{K_{m}}$. The number of modes $K_{m}$ identified with AOMA may vary from dataset to dataset. The algorithm will work with any general AOMA procedure. Here we use a data-driven Stochastic Subspace Identification (SSI) method together with a fully automated stabilisation-based procedure similar to the one proposed by Reynders et al. [6].

Before the AOMA data are processed they have to be randomly shuffled on the dataset scale. This will allow to account for sudden changes of OEC which otherwise may be identified as separate modes. In the case of the present dataset shuffling is required since only stepwise operational changes were measured. For datasets with only continuous OEV shuffling is not required.

\subsubsection{Find nearest neighbours from consecutive datasets}

Start with the first baseline dataset and calculate the distances between each mode $\lambda_{m, i}, \phi_{m, i}$ in the current dataset and all modes in the subsequent dataset $\lambda_{m+1, j}, \phi_{m+1, j}$. The distance between two modes can be measured in a variety of ways, here the sum of normalized pole distance and MAC is used:

$$
d p M A C_{i, j}=\frac{\left|\lambda_{i}-\lambda_{j}\right|}{\max \left(\left|\lambda_{i}\right|,\left|\lambda_{j}\right|\right)}+\left(1-M A C_{i, j}\right)
$$


The goal in this step is to find the nearest neighbour from dataset $m+1$ for each mode in dataset $m$. The neighbours for the last dataset $M$ are found from the first dataset. Hence, the final result of the procedure is a single nearest neighbour for each mode from each dataset and the dpMAC distance between the two.

Consistently identified modes will have nearest neighbours in near proximity to them, whereas modes that are not consistently identified, false identifications or modes that are only identified at certain OEC will tend to have significantly larger distances to the nearest neighbour.

To achieve finer separation of the modes for subsequent analysis a vector of multiple distance measures between the nearest neighbours according to Eq. (5) is created:

$$
\boldsymbol{p}_{\boldsymbol{i}}=\left[d \lambda_{i, j} d f_{u i, j} d \xi_{i, j}\left(1-M A C_{i, j}\right) d M P D_{i, j}\right]^{T}
$$

The mode distance vector $\boldsymbol{p}$ consists of the relative eigenvalue difference $d \lambda$, the relative natural frequency difference $d f$, the relative damping ratio difference $d \xi$, the Modal Assurance Criterion $M A C$ and the Mean Phase Deviation $M P D$. These properties were chosen since they all represent relative distance measures between modes from consecutive datasets. Together they include information from all extracted modal properties (natural frequencies, damping ratios and mode shapes). The individual features in Eq. (6) all resemble similar probability distributions driven by the nearest-neighbour detection procedure. Similar probability distributions are important for the subsequent feature transformation described in the next section.

\subsubsection{Separate observations into a low-distance and large-distance neighbours}

The nearest-neighbour distances in Eq. (6) can be used to separate all baseline modal observations into a small-distances and a large-distances cluster. The small-distances cluster congregates modes which are consistently identified throughout the whole baseline dataset. To separate the modes into these two sets two challenges have to be overcome: First, the nearest-neighbor detection process results in heavily skewed feature distributions. Classic clustering methods like k-means or Gaussian Mixture Model (GMM) expect the data to be normally distributed. Second, the procedure must be able to adapt in case that the small-distances cluster is significantly larger than the large-distances cluster.

The first challenge can be overcome by proper transformation and normalisation of the distance vector $\boldsymbol{p}$. Therefore, the distance vector (Eq. (6)) is transformed into a shape that more resembles a normal distribution. The power transformation is done according to Eq. (7) using the approach described by Box and Cox [24].

$$
h_{T, i}(m)= \begin{cases}\left(p_{i}^{\gamma_{m}}(m)-1\right) \cdot \gamma_{m}^{-1} & , \gamma_{m} \neq 0 \\ \ln \left(p_{i}(m)\right) & , \gamma_{m}=0\end{cases}
$$

The optimal transformation parameter $\gamma_{m}$ for each individual feature variable $p_{i}(m)$ is found by a profile log-likelihood maximisation.

Features with large dispersion will dominate the clustering process [25]. To give every variable equal weight the distance vector is normalized to standard scores using the standard deviation $\sigma\left(h_{T, i}(m)\right)$ and the mean $\bar{h}_{T, i}(m)$ of the individual (transformed) features:

$$
h_{N, i}(m)=\left(h_{T, i}(m)-\bar{h}_{T, i}(m)\right) / \sigma\left(h_{T, i}(m)\right)
$$

Equation (8) shows the final form of the transformed and normalised distance vector $\boldsymbol{h}_{\boldsymbol{N}}$. To overcome the challenge of dissimilar size clusters the two-cluster GMM model shown in Eq. (9) is fit to the data using Expectation-Maximization (EM) [26]. 


$$
\begin{aligned}
p\left(\boldsymbol{h}_{\boldsymbol{N}}\right)= & \sum_{k=1}^{2} \pi_{k} \mathcal{N}\left(\boldsymbol{h}_{\boldsymbol{N}} \mid \boldsymbol{\mu}_{\boldsymbol{k}}, \boldsymbol{\Sigma}_{\boldsymbol{k}}\right) \\
& \text { where } 0<\pi_{k}<1, \sum_{k=1}^{2} \pi_{k}=1
\end{aligned}
$$

The distance vector $\boldsymbol{h}_{N}$ is normalised to standard scores and it is assumed that that there are significantly more low-distance neighbours than large-distance neighbours. Therefore, the cluster centroids $\boldsymbol{\mu}_{\mathbf{1}}$ and $\boldsymbol{\mu}_{\mathbf{2}}$ are initialized with $\mathbf{- 1}$ and $+\mathbf{2}$ respectively. Furthermore, $\pi_{1}=0.9$ and $\pi_{2}=0.1$ are used as initial weights. The transformation according to Eq. (7) will stretch the low-distance range and compress the large-distance range. Hence, the covariance matrices $\boldsymbol{\Sigma}_{\mathbf{1}}$ and $\boldsymbol{\Sigma}_{\mathbf{2}}$ are initialized using $\boldsymbol{I}$ and $0.1 \cdot \boldsymbol{I}$ respectively, where $\boldsymbol{I}$ is the identity matrix. The result of the clustering procedure will be two sets $S_{1}$ and $S_{2}$ that contain the modes with low-distance neighbours and large-distance neighbours.

\subsubsection{Separate observations into consistent sets using hierarchical clustering}

The next step is to separate the individual modes from all baseline datasets into consistent clusters that each represent one physical mode of the system. Agglomerative hierarchical clustering is the most popular approach for tasks where the final number of clusters is not known beforehand [25]. All agglomerative hierarchical clustering procedures can be described in three steps:

1. Each observation starts in its own cluster.

2. The two nearest clusters are combined into a new cluster.

3. The procedure is repeated until all observations are contained in a single cluster.

For the issue at hand the procedure is stopped when the inter-cluster distance between two nearest clusters exceeds a certain distance threshold. The distance between the clusters is measured according to Eq. (5) and the threshold is derived from the dpMAC Probability Density Function (PDF) estimate of the low-distance set $S_{1}$. The threshold $\tilde{d}_{d p M A C}$ is chosen at the 99 th percentile of a Weibull distribution fitted to the $S_{1}$ subset of the data:

$$
P\left(d p M A C_{S_{1}} \leq \tilde{d}_{d p M A C}\right)=0.99
$$

Inter-clusters distances are calculated using average linkage

$$
d_{r, s}=\frac{1}{n_{r} n_{s}} \sum_{i=1}^{n_{r}} \sum_{j=1}^{n_{s}} \operatorname{dist}\left(x_{r i}, x_{s j}\right)
$$

where dist is the distance function according to Eq. (5), $n_{r}$ and $n_{s}$ are the total number of individual members $x_{r}$ and $x_{s}$ in the clusters $r$ and $s$ respectively. The procedure returns $M_{t}$ sets $S_{h c, 1} \ldots S_{h c, M_{t}}$ that contain varying number of observations. The observations in each individual cluster $S_{h c, m}$ are homogeneous according to Eq. (5).

Hierarchical clustering in combination with a sophisticated distance measure is computationally expensive. It is possible to significantly decrease the computational effort by more than an order of magnitude without altering the outcome of the procedure. The basic idea is to pre-cluster the whole group of baseline modes into smaller sub-clusters using a simple distance measure. The sub-clusters are then examined individually in a subsequent clustering step using the procedure described above. We use the pole distance $\mathrm{d} \lambda$ as the pre-cluster similarity measure and choose the cutoff distance $\tilde{d}_{d \lambda}$ to include $99.9 \%$ of all low-distance neighbours in $S_{1}$ according to the fitted probability distribution. 


\subsubsection{Outlier rejection}

In the next step an outlier rejection technique is used to remove observations with abnormal natural frequencies and damping ratios from every set $S_{h c, m}$. The sets $S_{h c, m}$ are homogeneous by means of Eq. (5). Hence, one could argue that outliers should have been removed during the hierarchical clustering procedure. However, the cutoff distance $\tilde{d}_{d p M A C}$ is derived from a mixture of nearest neighbour distances from different physical modes. Furthermore, Eq. (5) is a sum of three properties with considerably different identification variances. Hence, outlier in one property may be hidden within the variance of another. This leads to unnecessarily strong variances of individual features and consequently to a lower damage detection rate.

The outlier removal procedure used in this study is the modified Thompson Tau technique [27]. The procedure considers only one outlier at a time and is repeated until no more outliers are found. The algorithm first looks for the observation with the largest absolute value deviation from the mean:

$$
\delta=\max \left(\left|X_{i}-\bar{X}\right|\right)
$$

In our case the dummy variable $X$ is either the natural frequency $f_{u}$ or the damping ratio $\xi$. In the next step the modified Thompson $\tau$ is calculated from the student's t PDF.

$$
\tau=\frac{t_{\alpha / 2} \cdot(n-1)}{\sqrt{n} \cdot \sqrt{n-2+t_{\alpha / 2}^{2}}}
$$

Here $n$ is the number of observations and $t_{\alpha / 2}$ is the critical student's $\mathrm{t}$ value, which is a function of the number of observations $n$ and the significance level $\alpha . t_{\alpha / 2}$ can be calculated from the inverse of student's t cumulative distribution function. $\alpha$ is set to 0.01 to limit the removal to strong outliers. The final step of the algorithm is to test whether the absolute value deviation is larger than $\tau$ multiplied by the standard deviation of $X$, in which case the data point is rejected (Eq. (14)). The algorithm is repeated, starting from Eq. (12), until no more outliers are found.

$$
\delta>\tau \cdot \sigma(X)
$$

\subsubsection{Feature vector preparation}

The clusters $S_{h c, m}$ returned after the hierarchical clustering step may contain multiple observations from the same dataset. This can happen when e.g. the AOMA algorithm identifies two modes with nearly identical modal properties from a single dataset. Since there cannot be two representations of the same feature in one row of a feature vector, these duplications have to be removed. Therefore, the dpMAC distances of all observations from a single dataset to the cluster centroid are calculated. All but the nearest duplication are removed from the cluster. The procedure is repeated for every cluster.

The number of observations in every cluster $S_{h c, m}$ will vary. Some clusters will contain observations from nearly every baseline dataset. Others will consist of very few observations. Finally, there may be modes that are not well excited, that show a significantly higher identification variance than the remaining modes, that are not consistently detected or that are only detected or not detected under certain OEC. These will only be detected in a certain proportion of the baseline data. Hence, there exists a trade-off between the number of modes that can be used for subsequent damage detection and the percentage of baseline datasets that are feature-complete, i.e. have an observation in every cluster $S_{h c, m}$. The ratio between the number of observation $M_{h c, m}$ in a cluster $S_{h c, m}$ and the total number of baseline datasets $M$ can be used as a threshold to control this trade-off.

$$
\vartheta \leq \frac{M_{h c, m}}{M}
$$

Only the $K_{f c}$ clusters with a minimum number of objects $\vartheta \cdot M$ are retained for subsequent analysis. 
The final step of the baseline data preparation procedure is to remove all datasets that do not have a representative in each remaining cluster. An alternative approach, which is not pursued here, would be to reconstruct the missing mode information from datasets where these modes were identified (missing value treatment). The number of feature-complete baseline datasets is $N \leq M_{h c, m}$. These $N$ datasets are used to extract the $p$ individual features for the feature vector $X$ that are used for subsequent damage detection. In this work natural frequencies and damping ratios are used as features. Hence, one row $x_{i}^{T}$ of the feature vector $\boldsymbol{X}$ is defined as

$$
x_{i}=\left[f_{u, i, 1}, \ldots, f_{u, i, K_{f c}}, \xi_{u, i, 1}, \ldots, \xi_{u, i, K_{f c}}\right]^{T}
$$

where $f_{u, i, 1}, \ldots, f_{u, i, K_{f c}}$ are the $K_{f c}$ natural frequencies extracted from the $i$ th feature-complete dataset. $\xi_{u, i, 1}, \ldots, \xi_{u, i, K_{f c}}$ are the damping ratios extracted from the same dataset. The individual steps of the baseline preparation procedure are summarized in figure 3 .

\subsection{Modal tracking}

The baseline preparation procedure returns $K_{f c}$ clusters of consistently identified modes. In SHM newly arriving measurements are compared to the baseline dataset or training model to examine whether the dataset represents an anomaly or not. Since the number of modes identified from the newly arriving dataset is again indeterminate and the presence of modes that were also selected for the baseline set is not guaranteed, a procedure is needed that matches modes identified from the new dataset with modes that were selected for the baseline dataset.

First, if the number of modes in the new dataset $M_{\text {new }}$ is smaller than the number of modes in the baseline $K_{f c}$, the dataset is skipped. This is a rare event, since the number of modes selected for the baseline is smaller than the mean number of identified modes. In the next step the distance according to Eq. (5) between each mean baseline mode $k$ and every mode in the new dataset $l$ is calculated. This results in a $K_{f c} \times M_{n e w}$ dpMAC distance matrix $\boldsymbol{D}_{M T}$, where $K_{f c} \leq M_{n e w}$. The mean values of the baseline poles are calculated according to

$$
\bar{\lambda}_{k}=\frac{1}{N} \sum_{i=1}^{N} \lambda_{k, i}
$$

where $\lambda_{k, i}$ is the pole from the $i$ th dataset in cluster $k$ and $\bar{\lambda}_{k}$ is the mean cluster pole. The mean cluster mode shape $\bar{\phi}_{k}$ is calculated according to

$$
\begin{aligned}
\mathbf{U S V}^{T} & =\left[\phi_{k, \mathbf{1}}, \ldots, \phi_{k, N}\right] \\
\bar{\phi}_{\boldsymbol{k}} & =\boldsymbol{U}[:, \mathbf{1}]
\end{aligned}
$$

where $\boldsymbol{U}[:, \mathbf{1}]$ is the first column vector of the unitary matrix $\boldsymbol{U}$, which in turn is calculated from the SVD of all mode shapes $\phi_{k, 1}, \ldots, \phi_{k, N}$ in cluster $k$. The affiliation between the pair of modes $\bar{\lambda}_{k}, \bar{\phi}_{k}$ and $\lambda_{\text {new }, l}, \phi_{\text {new }, l}$ with the smallest dpMAC distance $D_{M T, k, l}$ is saved. The corresponding row $k$ and column $l$ are removed from the distance matrix $\boldsymbol{D}_{M T}$. The procedure is repeated until a mode from the new dataset was associated with every baseline cluster. If the number of new mode candidates is equal to or larger than the number of baseline modes, modal tracking will always be successful. However, this does note necessarily mean that a fitting mode candidate is guaranteed to be found. If the new dataset does not include an appropriate mode candidate for one or more baseline mode(s), the least unfitting candidate(s) will be chosen. This will almost certainly result in an outlier classification of such datasets in the subsequent anomaly detection step.

\subsection{Data normalization and damage detection}

The consideration of OEV is often called data normalization in the context of SHM. Multiple approaches to account for OEV are investigated in this work. First, the detectability of damage without further 
Shuffle $M$ AOMA datasets, each with $K_{m}$ modes $\lambda_{1} \ldots \lambda_{K_{m}}, \phi_{1} \ldots \phi_{K_{m}}$

Find nearest neighbour from next dataset according to $d p M A C$ for each mode $\lambda_{n, m}$, $\phi_{n, m}$ with $n=1 \ldots K_{m}, m=1 \ldots M$

For each pair of nearest neighbours calculate mode distance vector $\boldsymbol{p}$

Transform and normalize $\boldsymbol{p}$ into $\boldsymbol{h}_{\boldsymbol{N}}$

Cluster modes into small-distance

$\left(S_{1}\right)$ and large-distance $\left(S_{2}\right)$ observations using $\boldsymbol{h}_{\boldsymbol{N}}$ and GMM

Derive $\tilde{d}_{d p M A C}$, the 99 th percentile of a Weibull distribution fitted to the $S_{1}$ observations

Group all modes into consistent sets $S_{h c, m}$ using hierarchical clustering with $\tilde{d}_{d p M A C}$ as stopping criteria

Remove natural frequency and damping ratio outlier in each set $S_{h c, m}$

Remove all but one representation of each dataset in every set $S_{h c, m}$

Retain only the $K_{f c}$ sets $S_{h c}$ that contain at least $\vartheta \cdot M$ observations

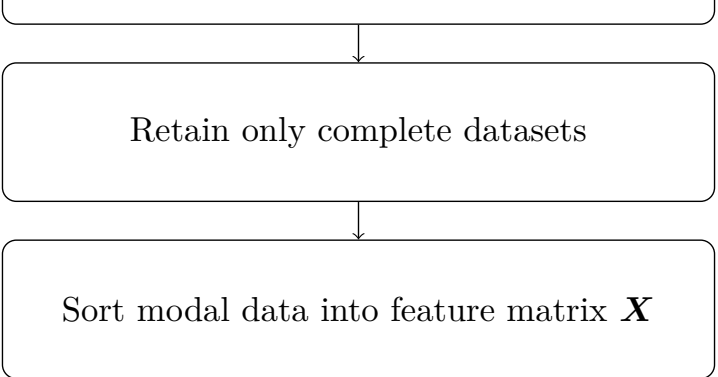

Figure 3: Summary of baseline preparation algorithm 
information about the currently encountered operational conditions is investigated. In this scenario anomalies are detected using the Mahalanobis Distance (MD), which is defined as

$$
d_{\text {mahal }}=\sqrt{\left(\boldsymbol{x}_{\text {new }}-\hat{\boldsymbol{\mu}}_{B L}\right)^{T} \hat{\boldsymbol{\Sigma}}_{\boldsymbol{B} \boldsymbol{L}}^{-1}\left(\boldsymbol{x}_{\text {new }}-\hat{\boldsymbol{\mu}}_{B L}\right)}
$$

where $\hat{\boldsymbol{\Sigma}}_{\boldsymbol{B} \boldsymbol{L}}$ is the covariance matrix estimate of the baseline feature vector $\boldsymbol{X}, \boldsymbol{x}_{\boldsymbol{n} \text { ew }}$ is the to be tested observation and $\hat{\boldsymbol{\mu}}_{\boldsymbol{B} \boldsymbol{L}}$ is the mean of each individual feature in $\boldsymbol{X}$. The direct application of the MD has been shown to be well-suited for damage detection under OEV [19, 28].

There are other popular approaches to account for unmeasured OEV, e.g. removal of the contribution from the major principal component before the application of Mahalanobis distance-based anomaly detection $[17,29,11]$. These approaches may result in higher damage sensitivity if the damage-induced feature vector changes point mainly into the directions of the minor components. However, this comes at the price that damage that manifests itself in a major principle component direction but outside the ellipsoid spanned by a Mahalanobis threshold cannot be detected using a minor PCA-based monitoring approach, whereas it could be detected if the Mahalanobis distance test were directly applied. Thus, since the feature vector changes induced by an arbitrary damage are not easily predictable and also vary depending on the currently encountered OEC (see table 1 and 2) PCA-based preprocessing is not used in this work.

To investigate whether the availability of direct measurements of the currently encountered OEV can substantially improve damage detectability, two approaches to incorporate this information are investigated. For the first approach the information about the currently encountered OEV is appended to the feature vectors

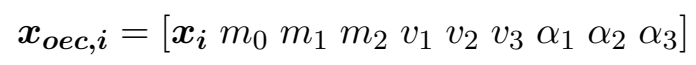

where $m_{0} \ldots \alpha_{3}$ are binary variables that indicate whether the specified OEC are currently encountered or not. For applications with continuously changing OEC these variables would be replaced by quantitative measurements of the OEV and normalised in the same manner as $\boldsymbol{x}_{\boldsymbol{i}}$. The second approach to consider OEV measurements is based on regression-based preprocessing and is commonly used in the AOMAbased SHM literature to account for direct measurements of the OEV [30,11]. Here the OEV is modelled using multiple output Linear Regression (LR)

$$
\boldsymbol{X}=\boldsymbol{O} \beta+\boldsymbol{E}
$$

where $\boldsymbol{X}$ is the $N \times p$ matrix of baseline features, $\boldsymbol{O}$ is the $N \times(m+1)$ matrix of binary variables that indicate whether the specified OEC are currently encountered or not, $\boldsymbol{\beta}$ is the $(m+1) \times p$ matrix of parameters and $\boldsymbol{E}$ is the $N \times p$ matrix of residuals [26]. For the regression-based approach the Mahalanobis test is applied to the residual matrix $\boldsymbol{E}$ instead of $\boldsymbol{X}$.

\section{Results and Discussion}

\subsection{Automated feature vector preparation}

Figure 4 shows a scatterplot of the transformed and normalized feature vector $\boldsymbol{h}_{\boldsymbol{N}}$, which was introdcued in Eq. (8). The small-distance and large-distance clusters $S_{1}$ and $S_{2}$ selected by the GMM (Eq. (9)) are represented with colors. The data show excellent separability in $\lambda$ and $f_{u}$, which are also strongly correlated. The clusters are not as well separable based on the other distance features. $\xi$ is known to 
show strong identification variance and the informative value in 1-MAC and dMPD is limited due to the use of only two sensors. Still, there is a significant amount of correlation present and the large-distance observations show a higher population density in the large-distance region of every investigated distance feature. It is important to point out that the large-distance observations are not directly removed as inconsistent. The GMM is only used to get a better estimate of what represents a consistently identified mode. This information is then used to derive a stopping criterion for the hierarchical clustering procedure.
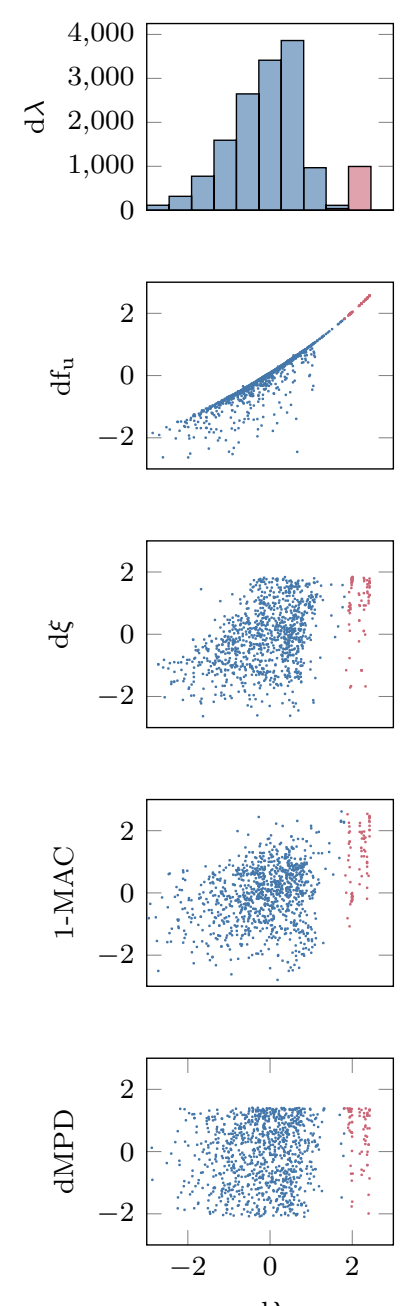

$\mathrm{d} \lambda$
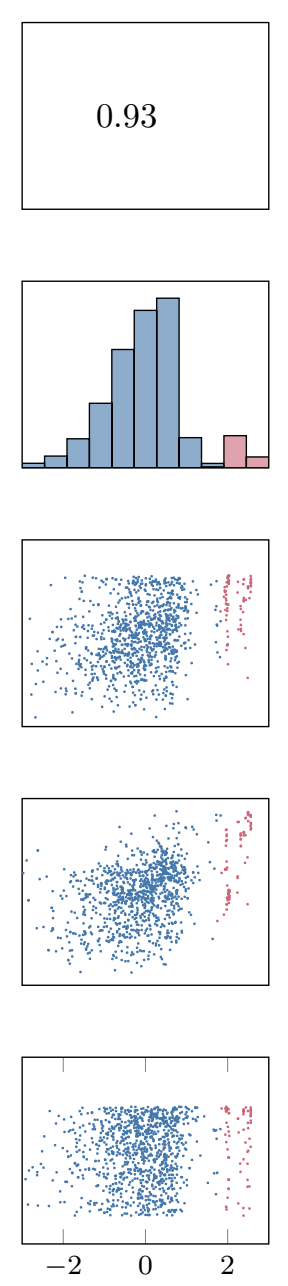

$\mathrm{df}_{\mathrm{u}}$
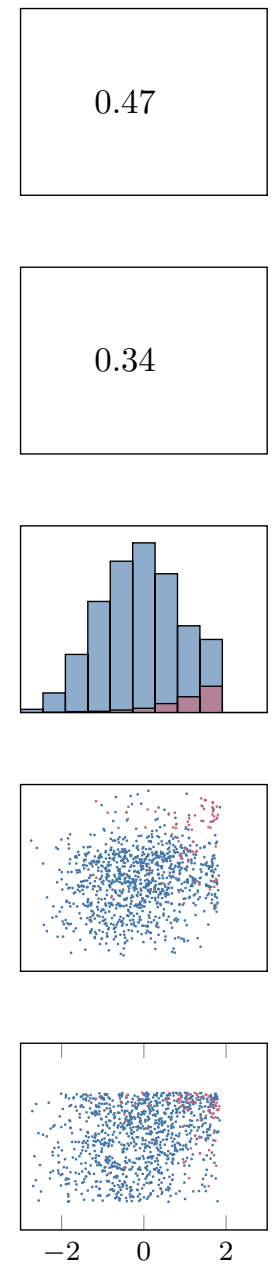

$\mathrm{d} \xi$
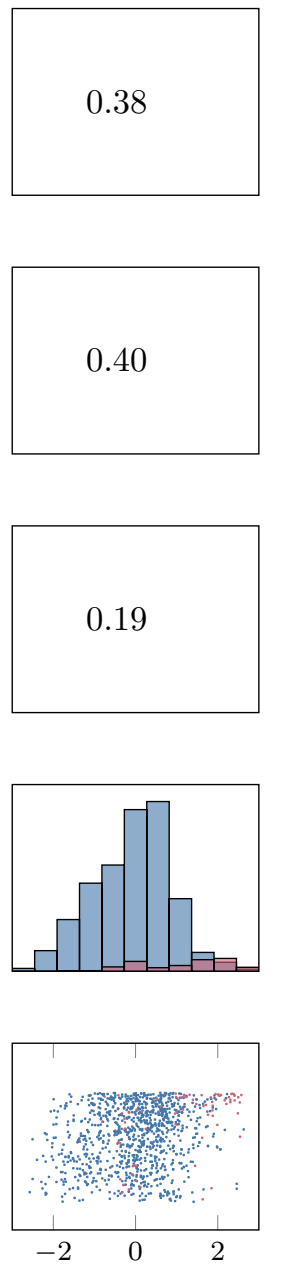

$1-\mathrm{MAC}$
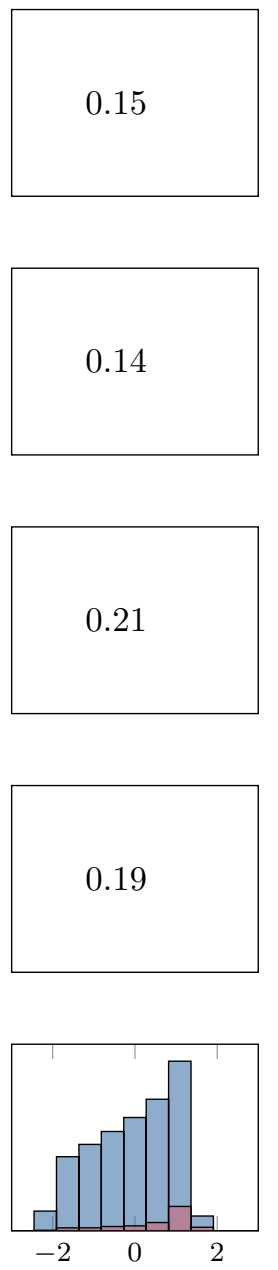

dMPD

$\square$ Small-distances cluster $\square$ Large-distances cluster

Figure 4: Scatterplot matrix of normalized distance vector $h_{N}$. The upper right triangle numbers are correlation coefficients between the individual feature variables. The number of shown observations was reduced for visualization purposes.

The investigated structure was equipped with only a single accelerometer and a single piezoelectric strain sensor. In order to obtain relevant consistency indicators from the MAC and the dMPD the modal properties were identified from the joined strain and accelerometer measurements. Thus, the MAC and dMPD in Eq. (5) and Eq. (6) are applied to a pattern of motion at the $k$ th equilibrium state where one component of the resulting mode shape represents an acceleration and the other represents a strain. The MAC (and dMPD) is used to measure the similarity of this pattern in observations from different datasets.

The vector in Eq. (6), shown in figure 4, contains two highly correlated features, namely $d \lambda$ and $d f_{u}$. Features with high correlation are known to create more "weight" in the clustering process [25], which in this case is a desired effect that is used to take into account that natural frequencies are excellent indicators of consistency and are known to be identified with significantly higher precision and accuracy than the other modal properties in OMA. Instead of scaling $d f_{u}$ to create more weight on that feature, $d \lambda$ is used to in addition capture the interaction between $d f_{u}$ and $d \xi$. Interaction-terms are commonly used 
in statistical learning to capture the interaction between variables and improve the overall performance of classification and clustering algorithms [26]. The dMPD feature shows comparatively little correlation with the other small-distance indicators and cannot be used to effectively distinguish between two nearly normal mode shapes, even if these are otherwise dissimilar. However, in aerospace applications the mode shapes are often markedly non-normal as a result of the fluid-structure interaction. Furthermore, large-distance observations can also be the result of false AOMA identifications, which may have an arbitrary amount of complexity. For the latter two scenarios the dMPD can be a valuable indicator of consistency.

The sensor setup investigated in this work consists of only two sensors. Thus, the informative value that can be extracted from the (mixed sensor) mode shapes is quite limited. It seems natural that measurements from more sensors will allow for better separation based on $p_{i}(4)$ (1-MAC) and $p_{i}(5)$ (dMPD) in Eq. (6). To verify this assumption, data from the FBGS shown in figure 1 were used. Due to the inherent limitations of the adopted Fiber Bragg Grating (FBG) interrogator [22] not enough modal data could be extracted from the measurements to allow for damage detection. However, under certain

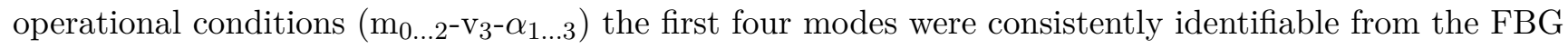
measurements. These data were used to investigate whether the correlation between the mode shape related and pole related features increases when all ten FBGS instead of only two of them are used to calculate $p_{i}(4)$ and $p_{i}(5)$. Applied to identical observations the correlation between all pole related quantities and all mode shape related quantities increased when ten sensors instead of two sensors were used, e.g. the correlation between $d \lambda$ and 1-MAC increased from 0.4 to 0.5 , the correlation between $d \lambda$ and dMPD increased from 0.21 to 0.35 . These results confirm that measurements at more Degrees of Freedom (DOFs) will improve the separation based on the mode shape related features.

One major objective during the development of the proposed methodology was to avoid arbitrarily chosen or user-defined parameters. Instead parameters are derived from the statistical properties of the present data. This, in principle, allows the algorithm to adapt to varying levels of noise, different inter-mode distances, variable number and type of sensors, etc. However, there are scenarios where the procedure can fail. If there exists no clear separation between the small-distance and the large-distance clusters (figure 4) GMM clustering may result in unpredictable partitioning. This can happen when the OEV induces modal parameter changes that are as far away from the cluster mean as a significant number of noise modes. However, due to the curse of dimensionality [26], this scenario is highly unlikely when a uniform distribution of noise modes is assumed and a multidimensional feature space is considered as it is the case here (Eq. (6)). A similar scenario could occur with two or more not consistently detected modes in near proximity to each other. In this work the procedure is shown to work with modal properties that were identified from only two sensors, which is at the lower end of the possible sensor quantity. More sensors will allow for better separation in the mode shape related dimensions $\left(p_{i}(4)\right.$ and $p_{i}(5)$ in Eq. (6)) rendering the two described scenarios even more unlikely.

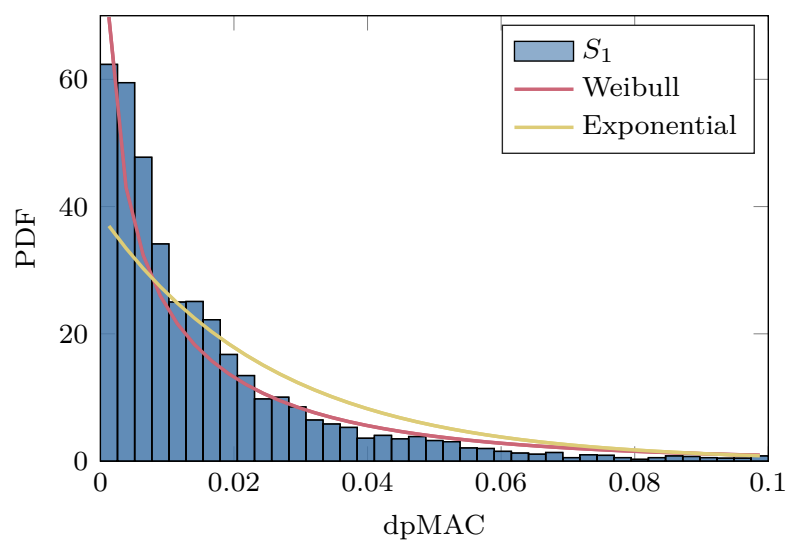

Figure 5: Small-distances cluster $S_{1}$ PDF distribution fitting.

Figure 5 shows the dpMAC small-distances observations distribution. A comparison of a large number of distributions from the exponential family showed that the Weibull distribution seems to best represent 
the nearest-neighbour detection procedure. Two fitted models are shown in figure 5 , an exponential and a Weibull distribution. The inverse cumulative distribution of the fitted model is used to calculate the 99th percentile $\tilde{d}_{d p M A C}$ (Eq. (10)), which is used as a stopping criterion for the hierarchical clustering procedure.

The process of hierarchical clustering is often visualised using a dendrogram, where the node height represents the distance at which two clusters are joined. A dendrogram, with the corresponding cutoff threshold according to Eq. (10), is shown in figure 6. The dendrogram shows a number of well separated clusters below the cutoff threshold. Their inter-cluster distances differ significantly. Some clusters are joined well below the threshold, whereas observations in other clusters are further apart from each other. The difference in in-cluster distances can be explained by different identification variances as well as by different sensitivities to OEV. This underlines one of the advantages of the approach proposed in this work. The cutoff distance automatically adapts to the level of variance in the baseline data. Instead of manual selection based on trial and error or experience for every individual use case an automatic and data-driven procedure is applied.

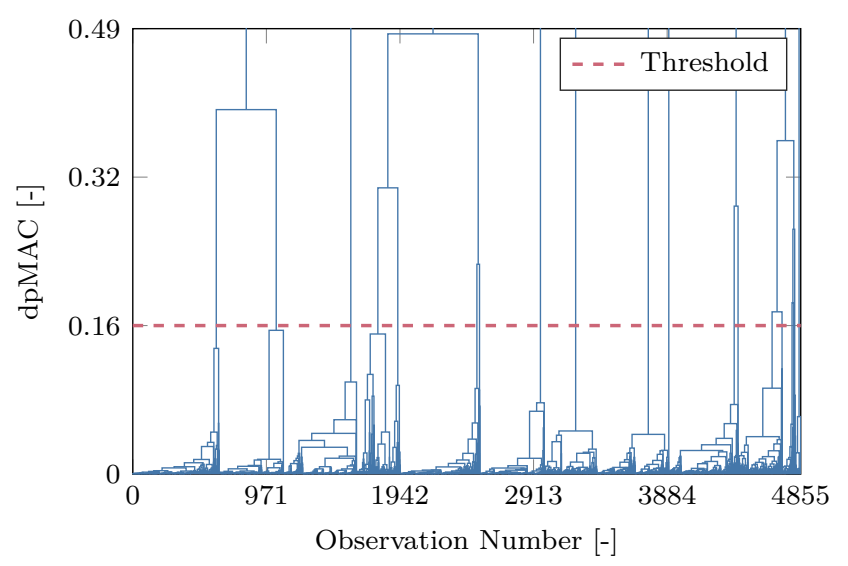

Figure 6: Hierarchical clustering dendrogram. The number of shown observations was reduced for visualization purposes.

The major disadvantage of AOMA-based SHM is that feature extraction is not guaranteed to be successful for every mode from every dataset. Some modes are identified with total consistence, whereas others are identified from for example $90 \%$ of all datasets. Hence, there exists a trade-off between the number of modes and the percentage of feature-complete datasets in the baseline dataset. More modes in the feature vector will generally increase the damage detection capabilities of the model and allow for better differentiation between various OEC and damage. However, the number of feature-complete datasets decreases when modes are included into the feature vector that are identified with lower than perfect consistency. Hence, the training set size decreases, resulting in worse model training performance and thus worse damage detection capability. Furthermore, there is no reason to assume that the percentage of feature-complete datasets will increase after the baseline has been built. Hence, depending on how missing features are handled either more datasets will be discarded during operation of the SHM system or the false alarm rate will increase. An effective procedure to reduce the false alarm rate without increasing the consistency threshold is discussed at the end of this section.

Figure 7 shows how the number of selected modes and the percentage of feature-complete datasets change when the required consistency is varied. Here the consistency threshold is defined as the ratio between the number of modes in a cluster returned by the automated baseline selection algorithm and the total number of initial baseline datasets $M$. The number of selected modes increases with decreasing consistency threshold. Only two modes are detected from every initial baseline dataset. In the range between $99 \%$ and $96 \% 5$ modes are selected. Two additional modes are selected in the range from $95 \%$ to $92 \%$. Simultaneously the percentage of feature-complete datasets decreases from $100 \%$ to $99 \%$ and then to $90 \%$. For subsequent analysis a threshold of $90 \%$ was chosen, which balanced the need to keep the threshold as high as possible whilst still capturing a sufficient number of modes. This threshold resulted in 8 selected modes and $82 \%$ feature-complete vectors. 


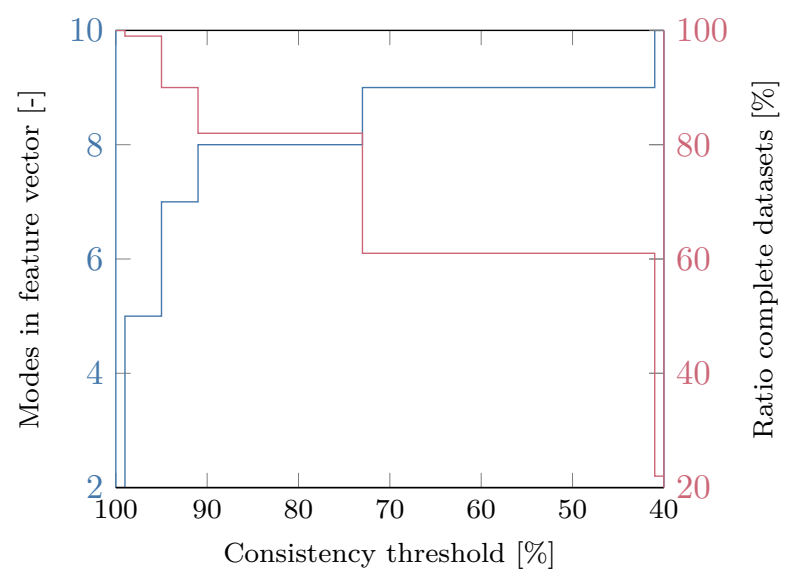

Figure 7: Relationship between the number of modes selected for the feature vector and the percentage of feature-complete baseline datasets.

Figure 8 illustrates the distance matrix $\boldsymbol{D}_{\boldsymbol{M} T}$ and one step of the modal tracking procedure outlined in section 2.4. The distance between each of the eight selected baseline modes and each new mode candidate is calculated using the dpMAC distance (Eq. (5)). In this case the test set contains eight mode candidates with complementing baseline clusters and three without. The three are dropped, whereas the eight are associated with their respective baseline clusters. The example shows that modes that are in near frequency proximity to each other are still well seperated according to the dpMAC distance. The distances between the new candidates and the baseline modes that belong together are at least an order of magnitude smaller than the distances to the neighbour modes. Since dpMAC contains information from three independent features (natural frequencies, damping ratios and mode shapes), the proposed procedure is immune to the problem of crossing frequencies. The example also shows that the smallest distances between neighbouring modes are approximately 0.3. Around this distance multiple clusters are joined in the dendrogram shown in figure 6. The hierarchical clustering cutoff distance lies between this distance and zero, the ideal distance values for two representations of the same physical mode at identical OEV. This further substantiates the well balanced automated choice of the cutoff distance.

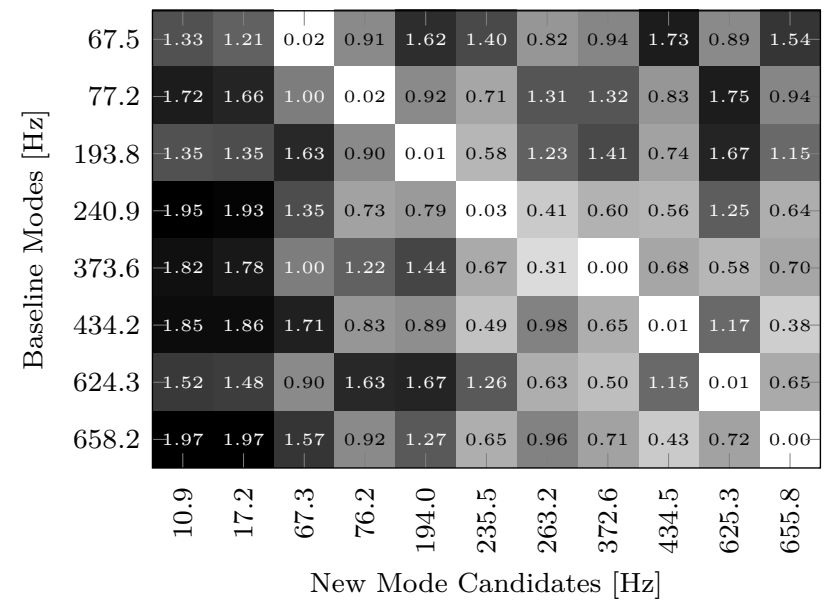

Figure 8: Modal tracking matrix with dpMAC values between baseline modes and new mode candidates.

Figure 9 shows the frequencies of all modes detected by AOMA from every investigated dataset. The region captioned "Baseline" was processed with the algorithm described in section 2.3. The regions "Test" and "Damaged" were prepared using the modal tracking algorithm described in section 2.4. The baseline and test sets were randomized during the automated baseline creation procedure, whereas the damaged set is ordered by the prevalent OEC. In accordance with the chosen consistency threshold of $90 \%$ eight modes were selected for the feature vector, which are highlighted using different colors. Two frequency regions with a high density of omitted modes are visible around $11 \mathrm{~Hz}$ and $260 \mathrm{~Hz}$. These 
are the first out-of-plane bending mode and the first in-plane bending mode, which are neglected since they are identified with a lower consistency than required by the chosen consistency threshold. Modes at $17 \mathrm{~Hz}, 35 \mathrm{~Hz}$ and $48 \mathrm{~Hz}$ were identified as narrow-banded wind tunnel-induced excitations, which are only detectable under certain OEC. The Baseline section shows a considerable number of omitted modes in near proximity to the eight selected feature vector modes. These are modes that were part of not feature-complete datasets. For a consistency threshold of $90 \%$ there proportion is approximately $18 \%$ (Fig. 7). Finally, a small number of randomly distributed modes are visible, which are the result of false AOMA identifications.

The proposed methodology relies on multiple statistical methods whose performance increases with the availability of more data. The approach was successfully tested and delivered consistent results for baseline observations from only three operation points (144 baseline datasets). The use of significantly less datasets may result in inconsistent behavior. To detect damage under operational variability many more samples of the normal state will usually be necessary. Multiple thousand samples are commonly used $[9,11,12]$. Thus, the proposed baseline preparation methodology does not require more datasets than one would actually need to set up an AOMA-based SHM system with manually selected and prepared features.

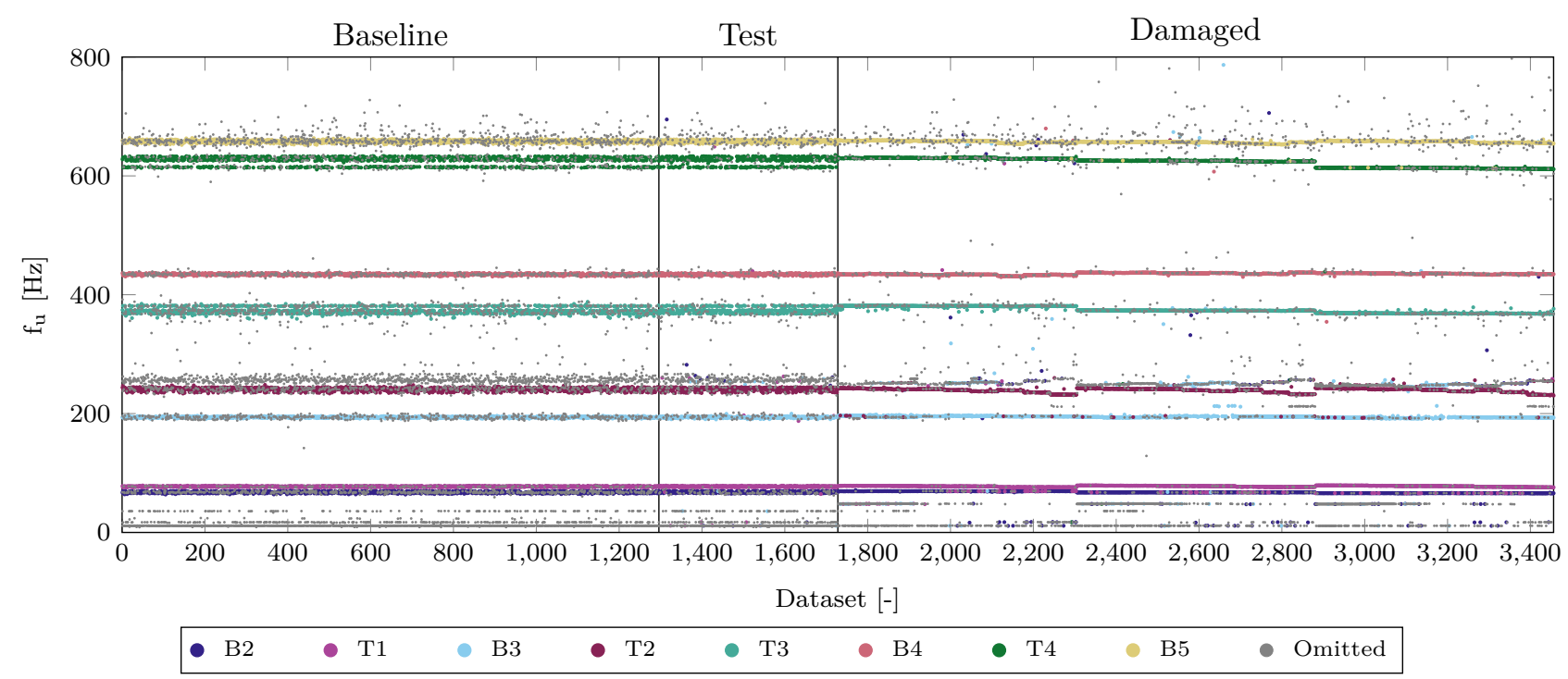

Figure 9: Natural frequencies identified by the AOMA algorithm from every investigated dataset. Representations of a single mode that were selected for the feature vector are highlighted with colors and denoted with their respective type of movement (where B stands for bending and $\mathrm{T}$ for torsion).

\subsection{Damage detection}

Figure 10 shows the natural frequencies and damping ratios of all eight feature vector modes at a single mass configuration $\left(\mathrm{m}_{0}\right)$. Frequencies and damping ratios clearly vary as a result of damage and OEV . The damage-induced frequency and damping ratio shifts are not constant and strongly depend on the present OEC. Table 1 shows the mean natural frequency shifts for all operation points together with the standard deviations at each of these operation points. Mass changes show the largest influence on the frequency with shifts up to 5\%. Velocity changes have a strong influence as well (over 3\%). The angle of attack changes still results in frequency shifts that are larger than 1\%. A comparison between the damaged and undamaged cases at otherwise constant operational conditions shows that the damaged-induced changes are well below $1 \%$ for the majority of operation points and modes (maximum shift $1.7 \%$ ). Table 2 shows damping ratio shifts as a result of OEV. The damping ratio shows a very strong sensitivity to damage and operational variability with shifts that range from $-67 \%$ to $+56 \%$ as a result of damage at otherwise constant OEC. The OEV-induced damping ratio shifts are even larger. However, it is evident from the standard deviations given in table 1 and table 2 that the damping ratio identification variability is in the same range as the changes to be detected and that the rather small 
damage (and OEC) sensitivity of the natural frequencies is outweighed by the excellent precision of the identification process. Still, the consideration of damping ratios as part of the feature vector results in a significantly improved damage missclassification rate on test data (-30\% to $-50 \%)$ and thus damping ratios are used as part of the feature vector throughout this study. Overall the dispersion of the individual damage sensitive features under OEV is much larger than the shifts introduced by the impact damage. Hence, the challenge in this study is to reliably identify a damage that produces feature changes that are significantly smaller than the (unmeasured) operational and environmental variability.

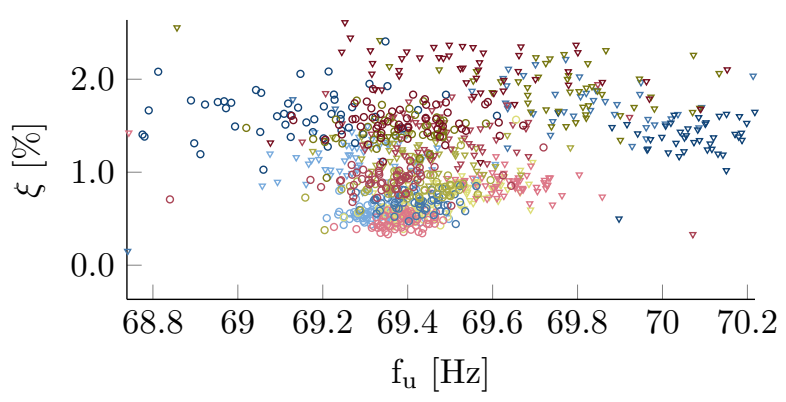

(a) B2

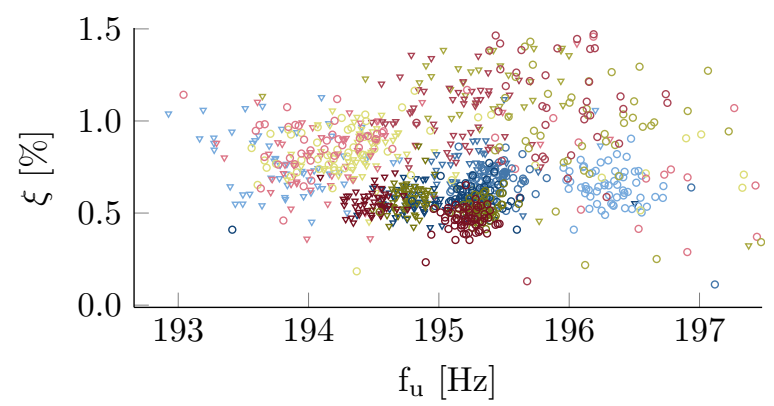

(c) B3

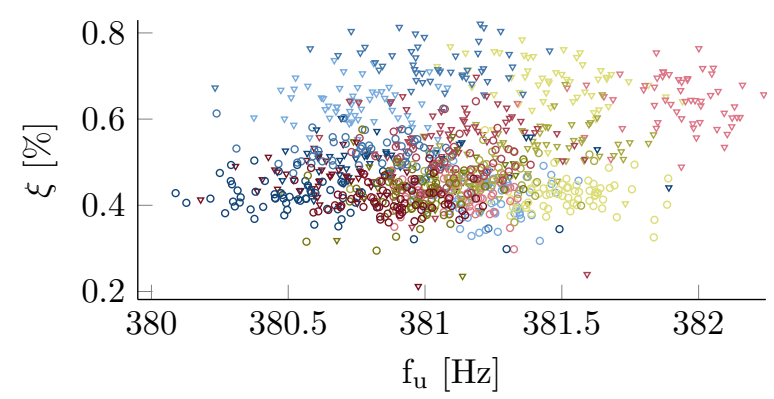

(e) $\mathrm{T} 3$

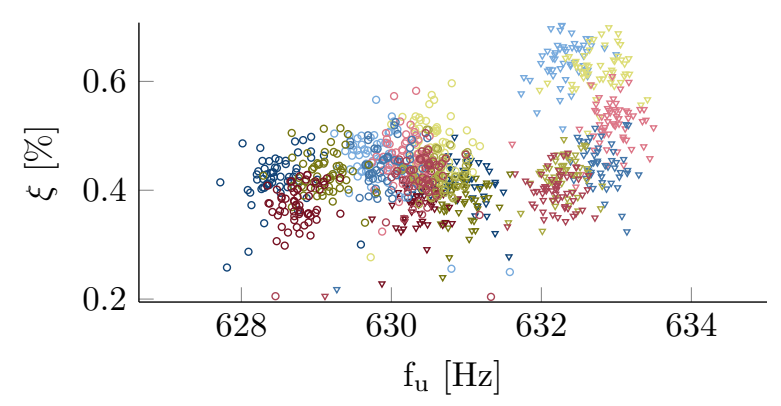

(g) $\mathrm{T} 4$

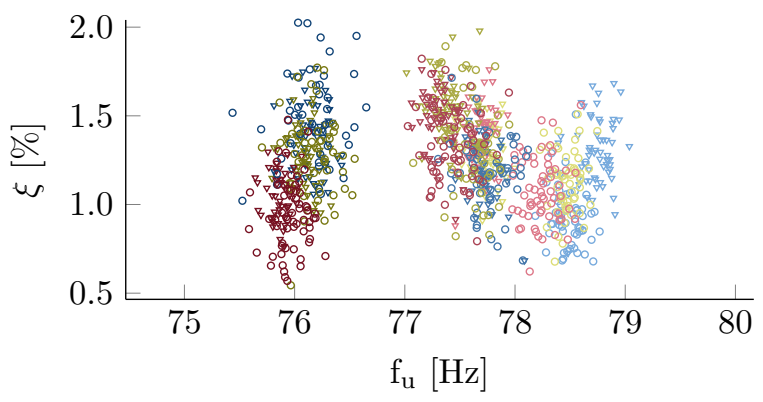

(b) $\mathrm{T} 1$

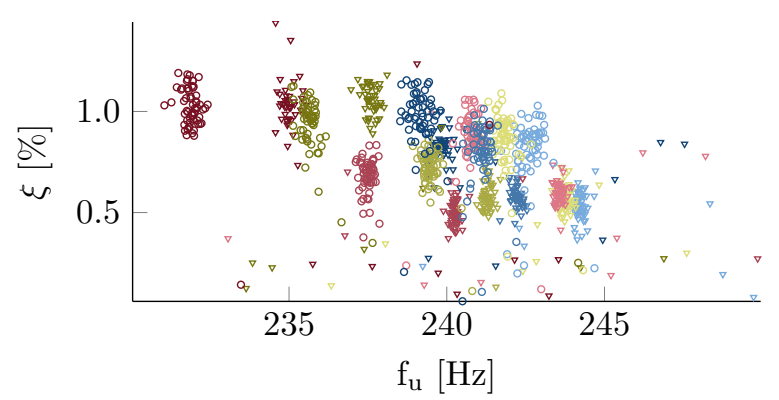

(d) $\mathrm{T} 2$

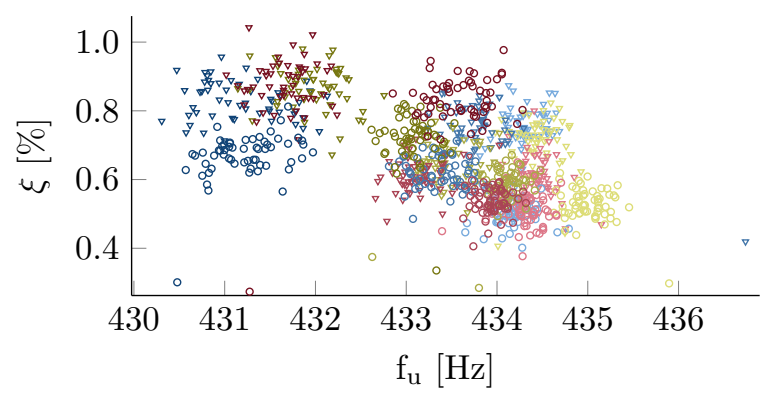

(f) B4

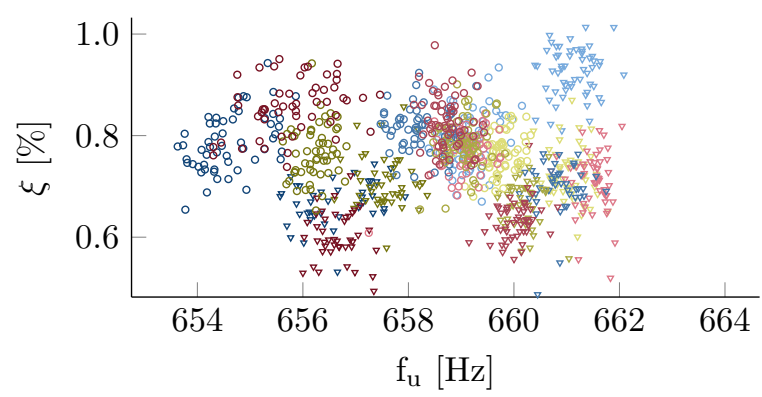

(h) B5

$\nabla \mathrm{d}_{0} / \mathrm{v}_{1} / \alpha_{1} \nabla \mathrm{d}_{0} / \mathrm{v}_{2} / \alpha_{1} \nabla \mathrm{d}_{0} / \mathrm{v}_{3} / \alpha_{1} \nabla \mathrm{d}_{0} / \mathrm{v}_{1} / \alpha_{2} \nabla \mathrm{d}_{0} / \mathrm{v}_{2} / \alpha_{2} \nabla \mathrm{d}_{0} / \mathrm{v}_{3} / \alpha_{2} \nabla \mathrm{d}_{0} / \mathrm{v}_{1} / \alpha_{3} \nabla \mathrm{d}_{0} / \mathrm{v}_{2} / \alpha_{3} \nabla \mathrm{d}_{0} / \mathrm{v}_{3} / \alpha_{3}$ $\circ \mathrm{d}_{1} / \mathrm{v}_{1} / \alpha_{1} \circ \mathrm{d}_{1} / \mathrm{v}_{2} / \alpha_{1} \circ \mathrm{d}_{1} / \mathrm{v}_{3} / \alpha_{1} \circ \mathrm{d}_{1} / \mathrm{v}_{1} / \alpha_{2} \circ \mathrm{d}_{1} / \mathrm{v}_{2} / \alpha_{2} \circ \mathrm{d}_{1} / \mathrm{v}_{3} / \alpha_{2} \circ \mathrm{d}_{1} / \mathrm{v}_{1} / \alpha_{3} \circ \mathrm{d}_{1} / \mathrm{v}_{2} / \alpha_{3} \circ \mathrm{d}_{1} / \mathrm{v}_{3} / \alpha_{3}$

Figure 10: Damping ratios $\xi$ over natural frequencies $\mathrm{f}_{\mathrm{u}}$ extracted from measurements at a constant mass configuration $\mathrm{m}_{0}$, where undamaged and damaged data $\left(\mathrm{d}_{0} / \mathrm{d}_{1}\right)$ are visualised through symbols, velocities $\left(\mathrm{v}_{1} / \mathrm{v}_{2} / \mathrm{v}_{3}\right)$ are visualised using saturation and angles of attack $\left(\alpha_{1} / \alpha_{2} / \alpha_{3}\right)$ by varying hue.

Figure 11 shows the results of the Mahalanobis distance-based anomaly detection. In figure 11a only 
Table 1: Natural frequency changes and standard deviations $\left(\bar{f}_{u} \pm \hat{\sigma}\right)$ due to the investigated damage d and OEV m, v and $\alpha$.

\begin{tabular}{|c|c|c|c|c|c|c|c|c|}
\hline $\mathrm{OEC}$ & $\begin{array}{c}\mathrm{B} 2 \\
{[\mathrm{~Hz}]}\end{array}$ & $\begin{array}{c}\mathrm{T} 1 \\
{[\mathrm{~Hz}]}\end{array}$ & $\begin{array}{c}\text { B3 } \\
{[\mathrm{Hz}]}\end{array}$ & $\begin{array}{c}\mathrm{T} 2 \\
{[\mathrm{~Hz}]}\end{array}$ & $\begin{array}{c}\mathrm{T} 3 \\
{[\mathrm{~Hz}]}\end{array}$ & $\begin{array}{c}\mathrm{B} 4 \\
{[\mathrm{~Hz}]}\end{array}$ & $\begin{array}{c}\mathrm{T} 4 \\
{[\mathrm{~Hz}]}\end{array}$ & $\begin{array}{c}\mathrm{B} 5 \\
{[\mathrm{~Hz}]}\end{array}$ \\
\hline $\mathrm{d} 0-\mathrm{m} 0-\mathrm{v} 1-\alpha 1$ & $69.28 \pm 0.33$ & $78.75 \pm 0.14$ & $194.0 \pm 0.7$ & $.2 \pm 1.2$ & $.9 \pm 3.3$ & $.3 \pm 0.2$ & $.3 \pm 0.3$ & \pm 0.4 \\
\hline $\mathrm{d} 0-\mathrm{m} 0-\mathrm{v} 1-\alpha 2$ & $69.50 \pm 0.29$ & $77.73 \pm 0.12$ & $194.4 \pm 0.3$ & $43.5 \pm 1.3$ & $1.3 \pm 1.9$ & $434.4 \pm 0.2$ & $632.8 \pm 0.3$ & $661.0 \pm 0.3$ \\
\hline $\mathrm{d} 0-\mathrm{m} 0-\mathrm{v} 1-\alpha 3$ & $69.50 \pm 0.56$ & $77.69 \pm 0.10$ & $194.2 \pm 0.5$ & $243.4 \pm 1.2$ & $381.5 \pm 1.4$ & $434.4 \pm 0.2$ & $633.0 \pm 0.3$ & $661.5 \pm 0.3$ \\
\hline $\mathrm{d} 0-\mathrm{m} 0-\mathrm{v} 2-\alpha 1$ & $69.15 \pm 1.07$ & $77.74 \pm 0.11$ & $195.2 \pm 0.6$ & $242.2 \pm 0.2$ & $380.1 \pm 3.3$ & $433.8 \pm 0.5$ & $632.8 \pm 0.2$ & $660.8 \pm 0.3$ \\
\hline $\mathrm{d} 0-\mathrm{m} 0-\mathrm{v} 2-\alpha 2$ & $68.75 \pm 0.94$ & $77.39 \pm 0.14$ & $194.8 \pm 1.3$ & $241.4 \pm 1.1$ & $380.9 \pm 2.1$ & $433.5 \pm 0.3$ & $632.2 \pm 0.8$ & $660.1 \pm 0.3$ \\
\hline $\mathrm{d} 0-\mathrm{m} 0-\mathrm{v} 2-\alpha 3$ & $68.91 \pm 0.93$ & $77.32 \pm 0.12$ & $195.1 \pm 0.6$ & $240.2 \pm 0.6$ & $380.8 \pm 1.4$ & $433.2 \pm 0.3$ & $632.2 \pm 0.2$ & $660.0 \pm 0.4$ \\
\hline $\mathrm{d} 0-\mathrm{m} 0-\mathrm{v} 3-\alpha 1$ & $69.98 \pm 1.06$ & $76.15 \pm 0.13$ & $194.8 \pm 0.3$ & $240.3 \pm 1.4$ & $379.3 \pm 3.6$ & $431.2 \pm 0.5$ & $630.9 \pm 0.3$ & $656.6 \pm 0.7$ \\
\hline $\mathrm{d} 0-\mathrm{m} 0-\mathrm{v} 3-\alpha 2$ & $69.87 \pm 0.32$ & $76.10 \pm 0.13$ & $194.8 \pm 0.1$ & $237.5 \pm 1.3$ & $380.0 \pm 3.9$ & $431.9 \pm 0.3$ & $630.9 \pm 0.5$ & $657.6 \pm 0.4$ \\
\hline $\mathrm{d} 0-\mathrm{m} 0-\mathrm{v} 3-\alpha 3$ & $69.46 \pm 0.54$ & $75.86 \pm 0.10$ & $194.5 \pm 0.1$ & $235.6 \pm 1.8$ & $378.9 \pm 4.2$ & $431.7 \pm 0.3$ & $630.4 \pm 0.2$ & $656.6 \pm 0.3$ \\
\hline $\mathrm{d} 0-\mathrm{m} 1-\mathrm{v} 1-\alpha 1$ & $67.48 \pm 0.28$ & $78.05 \pm 0.15$ & $3.9 \pm 0.3$ & $243.5 \pm 1.6$ & $374.0 \pm 1.6$ & $436.2 \pm 0.2$ & $627.7 \pm 0.2$ & $658.8 \pm 0.3$ \\
\hline $\mathrm{d} 0-\mathrm{m} 1-\mathrm{v} 1-\alpha 2$ & $67.52 \pm 0.40$ & $77.95 \pm 0.09$ & $193.7 \pm 0.5$ & $243.2 \pm 1.1$ & $373.1 \pm 2.9$ & $436.5 \pm 0.2$ & $626.9 \pm 0.8$ & $658.1 \pm 0.3$ \\
\hline $\mathrm{d} 0-\mathrm{m} 1-\mathrm{v} 1-\alpha 3$ & $67.73 \pm 0.14$ & $77.90 \pm 0.10$ & $194.1 \pm 0.3$ & $243.3 \pm 1.3$ & $373.2 \pm 1.8$ & $436.4 \pm 0.2$ & $627.4 \pm 0.2$ & $659.2 \pm 0.3$ \\
\hline $\mathrm{d} 0-\mathrm{m} 1-\mathrm{v} 2-\alpha 1$ & $67.22 \pm 0.59$ & $77.85 \pm 0.13$ & $193.9 \pm 1.6$ & $241.7 \pm 1.2$ & $373.2 \pm 0.9$ & $434.7 \pm 0.2$ & $626.7 \pm 0.2$ & $657.0 \pm 0.3$ \\
\hline $\mathrm{d} 0-\mathrm{m} 1-\mathrm{v} 2-\alpha 2$ & $67.20 \pm 0.52$ & $77.47 \pm 0.15$ & $4.4 \pm 0.5$ & $1.0 \pm 1.1$ & $373.0 \pm 1.9$ & $434.4 \pm 0.3$ & $626.3 \pm 0.2$ & $656.3 \pm 0.3$ \\
\hline $\mathrm{d} 0-\mathrm{m} 1-\mathrm{v} 2-\alpha 3$ & $67.27 \pm$ & $77.51 \pm 0.13$ & $194.1 \pm 1.1$ & $240.4 \pm 1.1$ & $372.6 \pm 2.0$ & $435.3 \pm 0.2$ & $627.0 \pm 0.2$ & $658.0=$ \\
\hline $\mathrm{d} 0-\mathrm{m} 1-\mathrm{v} 3-\alpha 1$ & $67.85 \pm 1.40$ & $76.28 \pm 0.14$ & $194.1 \pm 0.3$ & $239.8 \pm 1.1$ & $372.4 \pm 1.7$ & $432.6 \pm 0.5$ & $625.5 \pm 0.3$ & $653.6 \pm 0.7$ \\
\hline $\mathrm{d} 0-\mathrm{m} 1-\mathrm{v} 3-\alpha 2$ & $67.75 \pm 0.47$ & $76.25 \pm 0.10$ & $194.0 \pm 0.1$ & $237.7 \pm 1.0$ & $372.3 \pm 2.1$ & $433.4 \pm 0.3$ & $625.4 \pm 0.2$ & $654.5 \pm 0.4$ \\
\hline d0-m1-v3- $\alpha 3$ & $67.08 \pm 0.82$ & $76.30 \pm 0.10$ & $194.1 \pm 0.1$ & $235.8 \pm 0.9$ & $372.7 \pm 1.6$ & $434.8 \pm 0.3$ & $625.7 \pm 0.2$ & $655.7 \pm 0.3$ \\
\hline $\mathrm{d} 0-\mathrm{m} 2-\mathrm{v} 1-\alpha 1$ & $65.88 \pm 0.36$ & $78.07 \pm 0.10$ & $192.6 \pm 0.5$ & $4.1 \pm 1.6$ & $368.6 \pm 1.6$ & $435.7=$ & $615.3 \pm 0.8$ & 660.0 \\
\hline $\mathrm{d} 0-\mathrm{m} 2-\mathrm{v} 1-\alpha 2$ & $65.93 \pm 0.46$ & $77.97 \pm 0.11$ & $192.7 \pm 0.6$ & $244.1 \pm 1.5$ & $368.4 \pm 2.0$ & $435.8 \pm 0.2$ & $614.8 \pm 0.9$ & $660.0 \pm 0.4$ \\
\hline $\mathrm{d} 0-\mathrm{m} 2-\mathrm{v} 1-\alpha 3$ & $65.97 \pm 0.48$ & $77.64 \pm 0.13$ & $192.8 \pm 0.7$ & $243.6 \pm 1.8$ & $368.6 \pm 1.4$ & $435.3 \pm 0.2$ & $615.3 \pm 0.8$ & $660.6 \pm 0.3$ \\
\hline $\mathrm{d} 0-\mathrm{m} 2-\mathrm{v} 2-\alpha 1$ & $66.28 \pm 0.53$ & $77.88 \pm 0.16$ & $192.9 \pm 1.5$ & $242.6 \pm 1.6$ & $369.2 \pm 1.7$ & $435.0 \pm 0.2$ & $614.8 \pm 0.2$ & $659.1 \pm 0.3$ \\
\hline $\mathrm{d} 0-\mathrm{m} 2-\mathrm{v} 2-\alpha 2$ & $66.08 \pm 0.56$ & $77.53 \pm 0.15$ & $193.3 \pm 0.6$ & $241.2 \pm 1.1$ & $368.1 \pm 1.1$ & $433.7 \pm 0.3$ & $614.6 \pm 0.3$ & $658.0 \pm 0.4$ \\
\hline $\mathrm{d} 0-\mathrm{m} 2-\mathrm{v} 2-\alpha 3$ & $66.01 \pm 0.49$ & $77.52 \pm 0.15$ & $193.2 \pm 0.7$ & $240.7 \pm 0.3$ & $368.0 \pm 2.0$ & $434.6 \pm 0.2$ & $615.2 \pm 0.2$ & 659.6 \\
\hline $\mathrm{d} 0-\mathrm{m} 2-\mathrm{v} 3-\alpha 1$ & $66.17 \pm 0.69$ & $76.39 \pm 0.11$ & $193.0 \pm 0.4$ & $239.9 \pm 0.7$ & $368.4 \pm 3.2$ & $432.1 \pm 0.3$ & $614.0 \pm 0.2$ & $655.1 \pm 0.3$ \\
\hline $\mathrm{d} 0-\mathrm{m} 2-\mathrm{v} 3-\alpha 2$ & $65.86 \pm 0.56$ & $76.36 \pm 0.11$ & $192.8 \pm 0.2$ & $237.9 \pm 0.8$ & $367.7 \pm 2.1$ & $433.0 \pm 0.3$ & $614.0 \pm 0.2$ & $655.9 \pm 0.4$ \\
\hline d0-m2-v3- $\alpha 3$ & $65.66 \pm 0.59$ & $76.36 \pm 0.11$ & $192.8 \pm 0.1$ & $235.8 \pm 0.5$ & $368.0 \pm 2.6$ & $434.2 \pm 0.4$ & $614.0 \pm 1.2$ & $657.2 \pm 0.4$ \\
\hline $\mathrm{d} 1-\mathrm{m} 0-\mathrm{v} 1-\alpha 1$ & $69.36 \pm 0.25$ & $78.49 \pm 0.12$ & $196.3 \pm 0.2$ & $242.6 \pm 0.4$ & $380.8 \pm 1.3$ & $434.1 \pm 0.3$ & $629.8 \pm 0.4$ & $659.1 \pm 0.4$ \\
\hline $\mathrm{d} 1-\mathrm{m} 0-\mathrm{v} 1-\alpha 2$ & $69.40 \pm 0.05$ & $78.47 \pm 0.11$ & $194.8 \pm 1.2$ & $241.8 \pm 0.4$ & $381.0 \pm 1.8$ & $435.0 \pm 0.2$ & $630.5 \pm 0.3$ & $659.7 \pm 0.3$ \\
\hline $\mathrm{d} 1-\mathrm{m} 0-\mathrm{v} 1-\alpha 3$ & $69.37 \pm 0.13$ & $78.24 \pm 0.15$ & $194.9 \pm 1.4$ & $240.8 \pm 0.5$ & $380.7 \pm 1.3$ & $434.3 \pm 0.2$ & $630.2 \pm 0.2$ & $659.0 \pm 0.4$ \\
\hline $\mathrm{d} 1-\mathrm{m} 0-\mathrm{v} 2-\alpha 1$ & $69.40 \pm 0.12$ & $77.77 \pm 0.20$ & $195.5 \pm 0.3$ & $241.1 \pm 0.3$ & $380.7 \pm 0.3$ & $433.4 \pm 0.3$ & $630.0 \pm 0.2$ & $658.2 \pm 0.5$ \\
\hline $\mathrm{d} 1-\mathrm{m} 0-\mathrm{v} 2-\alpha 2$ & $69.41 \pm 0.09$ & $77.61 \pm 0.18$ & $196.2 \pm 0.5$ & $239.5 \pm 0.3$ & $380.6 \pm 1.7$ & $434.1 \pm 0.2$ & $630.6 \pm 0.2$ & $659.0 \pm 0.3$ \\
\hline $\mathrm{d} 1-\mathrm{m} 0-\mathrm{v} 2-\alpha 3$ & $69.29 \pm 0.23$ & $77.44 \pm 0.20$ & $195.9 \pm 0.4$ & $237.6 \pm 0.5$ & $380.7 \pm 1.7$ & $433.9 \pm 0.1$ & $630.4 \pm 0.4$ & $658.7 \pm 0.3$ \\
\hline $\mathrm{d} 1-\mathrm{m} 0-\mathrm{v} 3-\alpha 1$ & $69.07 \pm 0.35$ & $76.14 \pm 0.27$ & $195.2 \pm 0.3$ & $239.2 \pm 0.4$ & $380.1 \pm 1.5$ & $431.2 \pm 0.4$ & $628.5 \pm 0.3$ & $654.6 \pm 0.6$ \\
\hline $\mathrm{d} 1-\mathrm{m} 0-\mathrm{v} 3-\alpha 2$ & $69.37 \pm 0.11$ & $76.19 \pm 0.18$ & $195.3 \pm 0.2$ & $235.8 \pm 1.2$ & $380.7 \pm 1.2$ & $433.1 \pm 0.2$ & $629.1 \pm 0.3$ & $656.2 \pm 0.3$ \\
\hline d1-m0-v3- $\alpha 3$ & $69.39 \pm 0.12$ & $75.97 \pm 0.14$ & $195.2 \pm 0.1$ & $232.1 \pm 1.2$ & $380.8 \pm 0.9$ & $433.6 \pm 0.4$ & $628.8 \pm 0.2$ & $655.8 \pm 0.7$ \\
\hline $\mathrm{d} 1-\mathrm{m} 1-\mathrm{v} 1-\alpha 1$ & $67.44 \pm 0.05$ & $78.90 \pm 0.11$ & $194.2 \pm 0.2$ & $242.7 \pm 0.5$ & $373.8 \pm 0.1$ & $437.2 \pm 0.2$ & $625.9 \pm 0.3$ & $657.4 \pm 0.3$ \\
\hline d1-m1-v1- $\alpha 2$ & $67.43 \pm 0.05$ & $78.69 \pm 0.08$ & $193.5 \pm 0.7$ & $241.7 \pm 0.3$ & $373.5 \pm 0.1$ & $436.8 \pm 0.2$ & $625.4 \pm 0.2$ & $656.4 \pm 0.3$ \\
\hline $\mathrm{d} 1-\mathrm{m} 1-\mathrm{v} 1-\alpha 3$ & $67.51 \pm 0.05$ & $78.62 \pm 0.10$ & $193.9 \pm 0.6$ & $241.2 \pm 0.2$ & $373.5 \pm 0.1$ & $437.1 \pm 0.2$ & $625.4 \pm 0.3$ & $657.0 \pm 0.3$ \\
\hline $\mathrm{d} 1-\mathrm{m} 1-\mathrm{v} 2-\alpha 1$ & $67.38 \pm 0.07$ & $78.23 \pm 0.14$ & $194.3 \pm 1.1$ & $241.4 \pm 0.9$ & $373.3 \pm 0.1$ & $436.3 \pm 0.2$ & $625.6 \pm 0.2$ & $657.2 \pm 0.3$ \\
\hline $\mathrm{d} 1-\mathrm{m} 1-\mathrm{v} 2-\alpha 2$ & $67.39 \pm 0.08$ & $78.03 \pm 0.12$ & $195.5 \pm 0.6$ & $239.2 \pm 0.2$ & $373.1 \pm 0.1$ & $436.0 \pm 0.2$ & $625.2 \pm 0.2$ & $656.5 \pm 0.4$ \\
\hline $\mathrm{d} 1-\mathrm{m} 1-\mathrm{v} 2-\alpha 3$ & $67.50 \pm 0.09$ & $77.79 \pm 0.11$ & $195.4 \pm 0.8$ & $237.7 \pm 0.2$ & $373.3 \pm 0.2$ & $436.6 \pm 0.2$ & $625.5 \pm 0.2$ & $656.1 \pm 0.3$ \\
\hline d1-m1-v3- $\alpha 1$ & $67.48 \pm 0.10$ & $76.83 \pm 0.17$ & $195.0 \pm 0.2$ & $239.9 \pm 1.1$ & $373.3 \pm 0.1$ & $435.0 \pm 0.3$ & $624.3 \pm 0.2$ & $654.5 \pm 0.4$ \\
\hline d1-m1-v3- $\alpha 2$ & $67.43 \pm 0.11$ & $76.44 \pm 0.15$ & $194.7 \pm 0.1$ & $235.7 \pm 0.4$ & $373.0 \pm 0.2$ & $435.1 \pm 0.3$ & $623.8 \pm 0.2$ & $653.5 \pm 0.5$ \\
\hline d1-m1-v3- $\alpha 3$ & $67.57 \pm 0.10$ & $76.55 \pm 0.10$ & $194.8 \pm 0.2$ & $232.8 \pm 1.7$ & $373.5 \pm 0.1$ & $437.3 \pm 0.2$ & $624.5 \pm 0.2$ & $656.4 \pm 0.4$ \\
\hline $\mathrm{d} 1-\mathrm{m} 2-\mathrm{v} 1-\alpha 1$ & $66.13 \pm 0.05$ & $78.97 \pm 0.10$ & $193.4 \pm 0.2$ & $243.2 \pm 1.2$ & $369.3 \pm 0.5$ & $436.5 \pm 0.1$ & $613.6 \pm 0.2$ & $659.0 \pm 0.3$ \\
\hline $\mathrm{d} 1-\mathrm{m} 2-\mathrm{v} 1-\alpha 2$ & $66.15 \pm 0.06$ & $78.88 \pm 0.09$ & $192.9 \pm 0.2$ & $242.6 \pm 1.6$ & $369.2 \pm 0.5$ & $436.4 \pm 0.2$ & $613.4 \pm 0.2$ & $658.8 \pm 0.3$ \\
\hline $\mathrm{d} 1-\mathrm{m} 2-\mathrm{v} 1-\alpha 3$ & $66.14 \pm 0.05$ & $78.56 \pm 0.10$ & $192.7 \pm 1.1$ & $241.2 \pm 1.6$ & $368.8 \pm 0.6$ & $435.8 \pm 0.2$ & $613.6 \pm 0.2$ & $658.9 \pm 0.3$ \\
\hline $\mathrm{d} 1-\mathrm{m} 2-\mathrm{v} 2-\alpha 1$ & $66.00 \pm 0.10$ & $78.05 \pm 0.15$ & $192.4 \pm 1.0$ & $242.0 \pm 1.5$ & $368.7 \pm 1.2$ & $435.0 \pm 0.3$ & $613.4 \pm 0.2$ & $657.5 \pm 0.3$ \\
\hline d1-m2-v2- $\alpha 2$ & $66.17 \pm 0.08$ & $78.07 \pm 0.12$ & $193.2 \pm 1.5$ & $239.8 \pm 0.2$ & $368.9 \pm 1.1$ & $435.7 \pm 0.7$ & $613.5 \pm 0.2$ & $658.2 \pm 0.2$ \\
\hline $\mathrm{d} 1-\mathrm{m} 2-\mathrm{v} 2-\alpha 3$ & $66.12 \pm 0.09$ & $77.64 \pm 0.15$ & $193.8 \pm 0.3$ & $237.7 \pm 1.6$ & $368.6 \pm 1.5$ & $435.2 \pm 0.3$ & $613.0 \pm 0.2$ & $657.8 \pm 0.4$ \\
\hline d1-m2-v3- $\alpha 1$ & $66.14 \pm 0.10$ & $76.79 \pm 0.16$ & $193.6 \pm 0.2$ & $239.7 \pm 0.9$ & $368.5 \pm 0.5$ & $433.9 \pm 0.3$ & $612.4 \pm 0.5$ & $655.2 \pm 0.4$ \\
\hline $\mathrm{d} 1-\mathrm{m} 2-\mathrm{v} 3-\alpha 2$ & $66.11 \pm 0.23$ & $76.51 \pm 0.14$ & $193.5 \pm 0.1$ & $236.0 \pm 0.3$ & $368.5 \pm 1.2$ & $434.7 \pm 0.3$ & $612.4 \pm 0.2$ & $656.0 \pm 0.4$ \\
\hline d1-m2-v3- $\alpha 3$ & $65.98 \pm 0.18$ & $75.98 \pm 0.14$ & $193.3 \pm 0.2$ & $231.6 \pm 0.6$ & $368.4 \pm 1.3$ & $435.0 \pm 0.4$ & $611.8 \pm 0.3$ & $655.5 \pm 0.6$ \\
\hline
\end{tabular}


Table 2: Damping ratio changes and standard deviations $(\bar{\xi} \pm \hat{\sigma})$ due to the investigated damage $\mathrm{d}$ and OEV $\mathrm{m}, \mathrm{v}$ and $\alpha$.

\begin{tabular}{|c|c|c|c|c|c|c|c|c|}
\hline OEC & $\begin{array}{l}\text { B2 } \\
{[\%]}\end{array}$ & $\begin{array}{c}\mathrm{T} 1 \\
{[\%]}\end{array}$ & $\begin{array}{l}\text { B3 } \\
{[\%]}\end{array}$ & $\begin{array}{c}\mathrm{T} 2 \\
{[\%]}\end{array}$ & $\begin{array}{c}\text { T3 } \\
{[\%]}\end{array}$ & $\begin{array}{c}\mathrm{B} 4 \\
{[\%]}\end{array}$ & $\begin{array}{c}\mathrm{T} 4 \\
{[\%]}\end{array}$ & $\begin{array}{l}\text { B5 } \\
{[\%]}\end{array}$ \\
\hline$\alpha 1$ & 19 & .17 & \pm 0.16 & $2 \pm 0.12$ & $6 \pm 0.19$ & 0.05 & $3 \pm 0.05$ & م $02+02+0$ \\
\hline & & & & & & & 7 & \\
\hline & 8 & $+6-1$ & 6 & $6 \pm$ & 6 & 6 & 4 & 06 \\
\hline & $37 \pm 0.60$ & $22 \pm 0.14$ & $3 \pm 0$ & $8 \pm 0$ & $4 \pm 0$ & $75 \pm$ & $44 \pm 0.04$ & $70 \pm 0$ \\
\hline $\mathrm{d} 0-\mathrm{m} 0-\mathrm{v} 2-\alpha 2$ & $02 \pm 0.42$ & $55 \pm 0.16$ & $20 \pm 0.25$ & $57 \pm 0.10$ & $52 \pm 0.08$ & $64 \pm$ & $.42 \pm 0.04$ & $0.67 \pm 0.05$ \\
\hline -v2- $\alpha 3$ & $25 \pm 0.42$ & & & \pm & $4 \pm$ & & $0 \pm$ & $63 \pm$ \\
\hline & 25 & 8 & $6 \pm$ & $9 \pm$ & $3 \pm$ & $3 \pm$ & $11 \pm$ & $65 \pm$ \\
\hline & \pm & $7 \pm$ & $8 \pm$ & $5 \pm$ & $9 \pm$ & $7 \pm$ & $8 \pm$ & $1 \pm$ \\
\hline $\mathrm{d} 0-\mathrm{m} 0-\mathrm{v} 3-\alpha 3$ & $07 \pm 0.31$ & $05 \pm 0$ & $6 \pm$ & $6 \pm 0.23$ & $39 \pm 0.15$ & $87 \pm 0.07$ & $37 \pm 0.03$ & $60 \pm 0.05$ \\
\hline $\mathrm{d} 0-\mathrm{m} 1-\mathrm{v} 1-\alpha 1$ & $08 \pm 0.17$ & $46 \pm 0.18$ & & $2 \pm 0.15$ & $57 \pm$ & $55 \pm$ & $58 \pm$ & $67 \pm$ \\
\hline & \pm & $8 \pm$ & & $2 \pm$ & $1 \pm$ & $7 \pm$ & $2 \pm$ & $3 \pm$ \\
\hline $1-v 1-\alpha 3$ & $6+6,-$ & $7+6,-5$ & 6 & $4 \pm$ & $4 \pm$ & 5 & $1 \pm$ & 05 \\
\hline $\mathrm{d} 0-\mathrm{m} 1-\mathrm{v} 2-\alpha 1$ & $02 \pm 0$ & $6 \pm$ & $7 \pm$ & $6 \pm 0$ & $3 \pm 0$ & $4 \pm$ & $18 \pm$ & $70 \pm$ \\
\hline $\mathrm{d} 0-\mathrm{m} 1-\mathrm{v} 2-\alpha 2$ & $111+8$ & $8+1,-1$ & 8 & 9 & 1 & 7 & $2 \pm$ & 4 \\
\hline $\mathrm{d} 0-\mathrm{m} 1-\mathrm{v} 2-\alpha 3$ & $29 \pm$ & $9 \pm$ & 8 & 0 & 6 & 5 & $4 \pm$ & $2 \perp$ \\
\hline $1-1$ & & 7 & & 6 & $7 \pm$ & & $1 \pm$ & $5 \pm$ \\
\hline & 1 & \pm & 0 & $7 \pm$ & $4 \pm$ & 6 & $9 \pm$ & $4 \pm$ \\
\hline & $1.96 \pm$ & $78+6-1$ & 6 & $5 \pm 0$ & $6 \pm$ & $35 \pm$ & $37 \pm$ & $88 \pm$ \\
\hline $2-v 1-\alpha 1$ & $13 \pm$ & $8 \pm$ & $77 \pm$ & $5 \pm 0$ & $3 \pm$ & $3=$ & $7 \pm$ & $8 \pm$ \\
\hline & \pm & \pm & $-2+2$ & $2 \pm$ & \pm & & $3 \pm$ & \pm \\
\hline & $1+$ & $7+$ & 9 & $7 \pm$ & \pm & & \pm & 5 \\
\hline$x 1$ & 0 & $6 \pm$ & 6 & 5 & $7 \pm$ & & $7 \pm$ & 04 \\
\hline $2-v 2-\alpha 2$ & $24+8>$ & $7+$ & 6 & 8 & 2 & 4 & 4 & \\
\hline$-v 2-\alpha 3$ & $38 \pm$ & $8 \pm$ & 6 & $1 \pm$ & 3 & 6 & $9 \pm$ & $5 \pm$ \\
\hline & & & & $1+$ & 5 & & 4 & $5+$ \\
\hline & 1 & & & 3 & 4 & & 5 & 05 \\
\hline & $2.02 \pm$ & $0 \pm$ & $6 \pm$ & $5 \pm$ & $9 \pm$ & 6 & $8 \pm$ & $50 \pm$ \\
\hline & $0.56+0$ & & & & & & & \\
\hline & & & & & 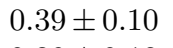 & & 5 & 4 \\
\hline & + & $8+$ & 3 & 6 & 2 & 5 & 4 & 04 \\
\hline & $7 \pm$ & $6 \pm$ & $7 \pm$ & 5 & 0 & & $4 \pm$ & $1 \pm$ \\
\hline & $0.83 \pm$ & $2 \pm$ & & & & & & \\
\hline$-\alpha 3$ & \pm & \pm & & 2 & 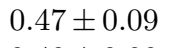 & & $2 \pm$ & 5 \\
\hline d1- & 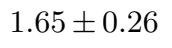 & $6+$ & 7 & 9 & 9 & 7 & 5 & 06 \\
\hline & & $7 \pm$ & 5 & 7 & & 7 & 4 & $6 \pm$ \\
\hline & $1.55 \pm 0$ & $0.94 \pm$ & $16 \pm$ & $1 \pm 0$ & $0 \pm$ & $3 \pm$ & $8 \pm$ & $5 \pm$ \\
\hline d1-m1-v1- $\alpha 1$ & $0.58 \pm 0$ & $02 \pm 0$ & 7 & $7 \pm 0$ & $10 \pm$ & $54 \pm$ & $0.47 \pm$ & $0.73 \pm$ \\
\hline & + & \pm & 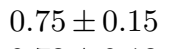 & \pm & \pm & 4 & $2 \pm$ & $3 \pm$ \\
\hline$\alpha 3$ & 8 & 3 & 3 & 3 & & 5 & 6 & 04 \\
\hline $\mathrm{d} 1-\mathrm{m} 1-\mathrm{v} 2-\alpha 1$ & $0.97 \pm 0.12$ & $60 \pm$ & $3 \pm$ & $0 \pm$ & $1 \pm$ & $7 \pm$ & $3 \pm$ & $82 \pm 0$ \\
\hline $\mathrm{d} 1-\mathrm{m} 1-\mathrm{v} 2-\alpha 2$ & $1.09 \pm 0.11$ & $46 \pm 0.18$ & & $1 \pm$ & & $68=$ & $39 \pm$ & $0.81 \pm 0$ \\
\hline$-v 2-\alpha 3$ & $0.83 \pm$ & $-a_{-1}$ & & 6 & $8 \pm$ & & $1 \pm$ & $1 \pm$ \\
\hline$-v 3-\alpha 1$ & $1.30 \pm$ & 1 & 9 & 4 & 4 & 3 & 4 & $5 \pm$ \\
\hline $1-v 3-\alpha 2$ & $1.33 \pm 0.16$ & $1 \pm$ & $4 \pm$ & $1 \pm 0$ & $1 \pm$ & $1 \pm$ & $0.39 \pm($ & $0.80 \pm 0.05$ \\
\hline$-v 3-\alpha 3$ & $1.48 \pm 0.16$ & $0.99 \pm 0.12$ & $52 \pm 0.08$ & $3 \pm 0.08$ & $10 \pm 0$ & $81 \pm$ & $0.36 \pm 0$ & $0.85 \pm 0.07$ \\
\hline & $0.58 \pm 0$ & $1.06 \pm$ & & & & & $0.50 \pm$ & \\
\hline $\mathrm{d} 1-\mathrm{m} 2-\mathrm{v} 1-\alpha 2$ & $0.55 \pm 0.08$ & $1.12 \pm$ & & & & & & \\
\hline $\mathrm{d} 1-\mathrm{m} 2-\mathrm{v} 1-\alpha 3$ & $0.48 \pm 0.07$ & $1.17 \pm 0$ & $0.83 \pm$ & $80 \pm 0.10$ & $8 \pm 0$ & $0.55 \pm$ & $0.48 \pm 0.04$ & $0.79 \pm 0.05$ \\
\hline $\mathrm{d} 1-\mathrm{m} 2-\mathrm{v} 2-\alpha 1$ & $0.87 \pm 0.16$ & $1.60 \pm 0.16$ & $0.98 \pm 0.19$ & $0.70 \pm 0.15$ & $0.37 \pm 0.09$ & $0.61 \pm 0.05$ & $0.45 \pm 0.03$ & $0.87 \pm 0.05$ \\
\hline $\mathrm{d} 1-\mathrm{m} 2-\mathrm{v} 2-\alpha 2$ & $0.82 \pm 0.12$ & $1.34 \pm 0.17$ & $11 \pm 0.36$ & $0.73 \pm 0$ & $0.40 \pm 0.13$ & $0.59 \pm$ & $0.45 \pm 0.04$ & $0.82 \pm 0.04$ \\
\hline $\mathrm{d} 1-\mathrm{m} 2-\mathrm{v} 2-\alpha 3$ & $0.82 \pm$ & $1.15 \pm$ & 15 & $0.69 \pm$ & & & & $0.77 \pm 0.04$ \\
\hline d1-m2-v3- $\alpha 1$ & $1.27 \pm 0.16$ & $1.71 \pm 0.20$ & $0.66 \pm$ & $0.86 \pm 0.12$ & $0.39 \pm 0$ & $0.64 \pm$ & $0.45 \pm 0.05$ & $0.87 \pm 0.05$ \\
\hline $\mathrm{d} 1-\mathrm{m} 2-\mathrm{v} 3-\alpha 2$ & $1.18 \pm 0.14$ & $1.38 \pm 0.17$ & $0.54 \pm 0.07$ & $0.95 \pm 0.12$ & $0.40 \pm 0.06$ & $0.66 \pm 0.04$ & $0.44 \pm 0.04$ & $0.91 \pm 0.05$ \\
\hline d1-m2-v3- $\alpha 3$ & $1.34 \pm 0.22$ & $1.01 \pm 0.12$ & $0.45 \pm 0.09$ & $1.04 \pm 0.16$ & $0.40 \pm 0.09$ & $0.71 \pm 0.06$ & $0.44 \pm 0.04$ & $0.82 \pm 0.05$ \\
\hline
\end{tabular}


data at a constant mass $\mathrm{m}_{0}$ are considered for training and damage detection. In figure $11 \mathrm{~b}$ the full range of measured operational variability is investigated. In figure 11c again full operational variability is considered. However, the information about the currently encountered OEC are appended to the feature vector. Results presented and discussed throughout this section are based on 5-fold cross-validation of the undamaged data. The diagrams in figure 11 each show one of those folds. All three diagrams show an accumulation of observations at the upper edge of the diagram from the Test and Damaged datasets. These observations are further away from the mean than six times the standard error estimate of the Mahalanobis distance in Baseline and were mapped to this value for visualisation purposes. These strong outliers come from datasets where at least one baseline mode was not detected in the corresponding Test or Damaged dataset. Identifications with not detected modes are not uniformly distributed over the different OEC. Instead, some modes show a significantly higher rate of missing modes.

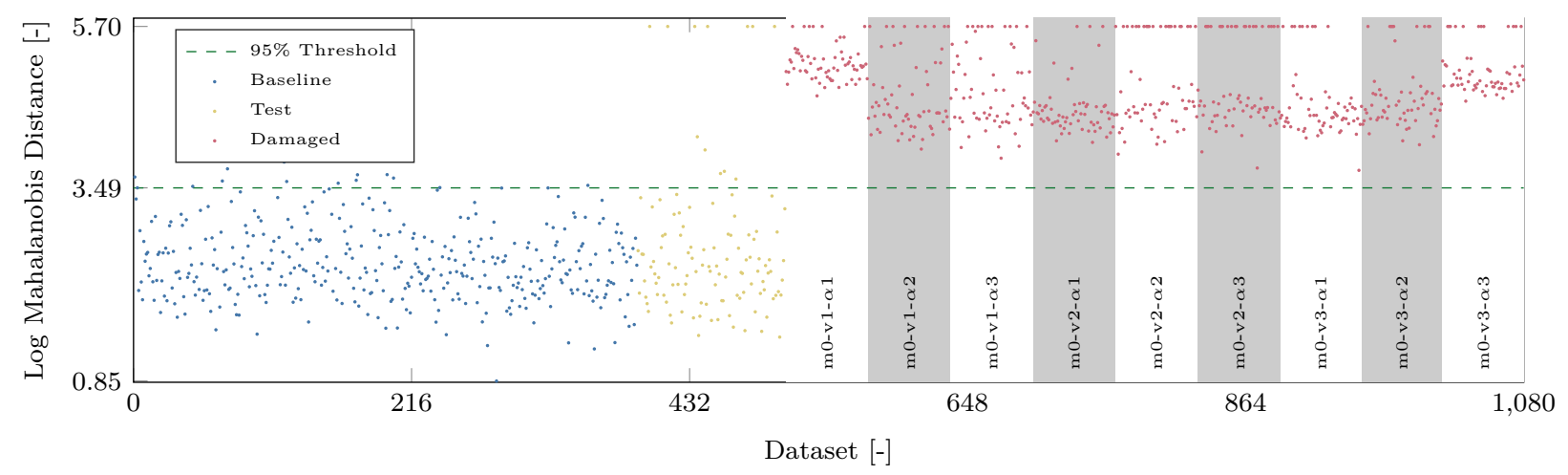

(a) Data at a single constant mass $m_{0}$ but otherwise unmeasured OEC.

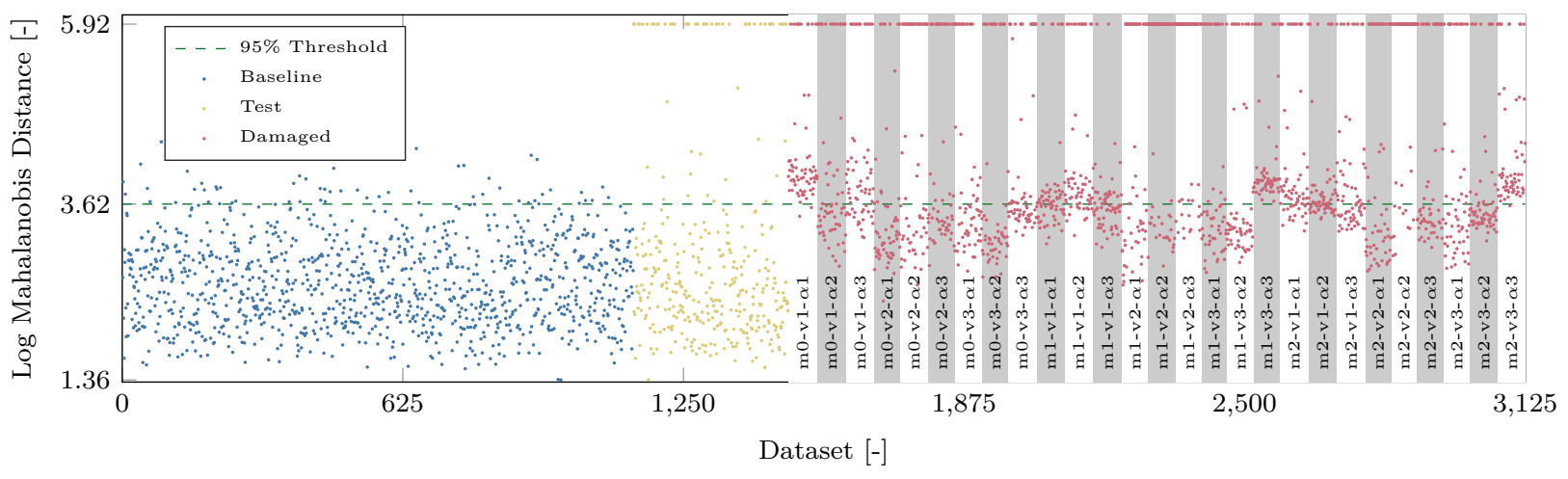

(b) Data with full operational variability and unmeasured OEC.

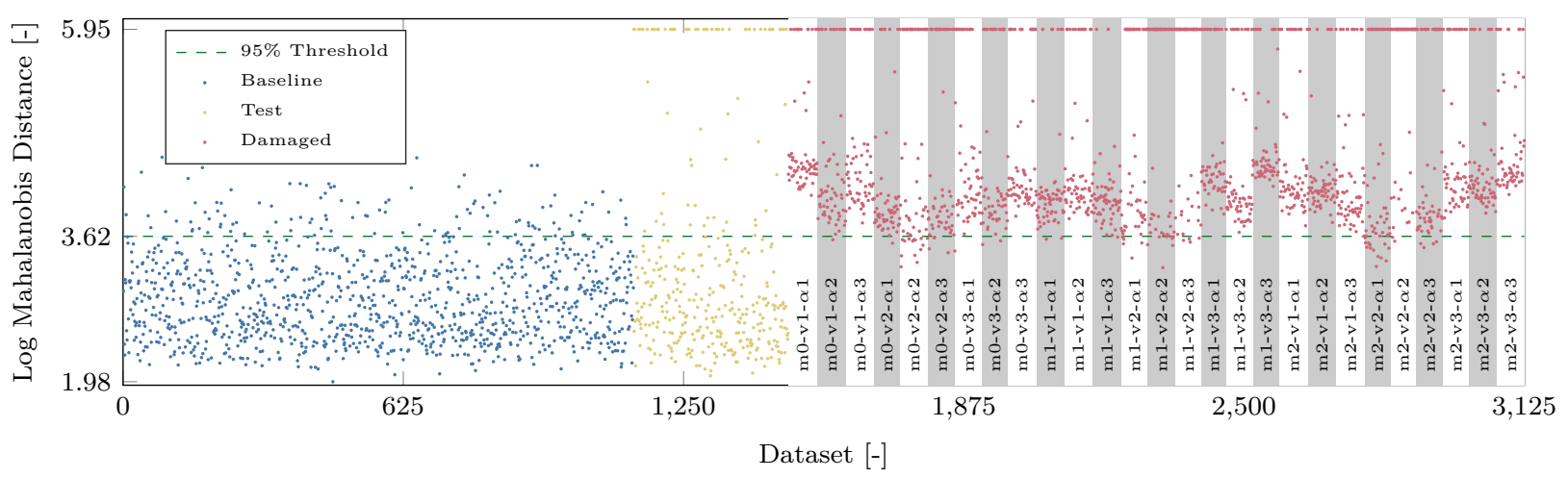

(c) Data with full operational variability and with OEC augmented feature vector.

Figure 11: Mahalanobis distance-based damage detection.

Figure 11a shows the excellent damage detection capabilities of the proposed methodology under varying wind-excitation, i.e. when only datasets at a constant mass are considered during training and for subsequent testing. The datasets used for this figure correspond to the data shown in figure 10. Hence, training is done under varying velocities and angles of attack. From the large Mahalanobis distance jump 
in the outlier class it is obvious that the target and the outlier class are strongly separated when the entire feature space is considered. This type of strong separability is not visible in the 16 individual features shown in figure 10. The excellent separability can be explained by the different impact of the damage and the wind-related variability on the dynamic properties of the specimen. The damage will result in a local modification of the specimen and its discrete stiffness and damping matrix approximation. The investigated wind-induced changes on the other hand result in more widely spread or global modifications of the stiffness and damping matrices [20]. Hence, feature vector changes introduced by local damage and global wind-excitation show into different directions. This is the key property that is necessary to make damage detection under OEV without explicit measurements of the variability feasible [17]. An investigation of the other two mass configurations show similar results.

The picture changes dramatically when the mass variability is introduced. Figure $11 \mathrm{~b}$ shows that a large proportion of Damaged samples falls below the $95 \%$ threshold and are therefore misclassified. The missclassification rate strongly depends on the currently encountered OECs. For some OECs damage is still reliably detectable. However, the overall missclassification rate would certainly be unacceptable for productive application. The mass changes introduce local modifications of the mass matrix, which result in feature vector changes that are more aligned with the damage-induced changes. Hence, local mass changes introduced in the vicinity of the damage location are hard to separate reliably from damage without explicit measurements of the OEC. Figure 11c shows the damage detection performance significantly improves when information about the currently encountered OEC are part of the feature vector. In this case each feature vector $\boldsymbol{x}_{\boldsymbol{i}}$ (Eq. (16)) is extended with OEC information according to Eq. (20). The provision of OEC information lifts the Damaged observations above the $95 \%$ threshold. However, damage is still at the verge of detectability for some OECs and the separation between the target and outlier class is not as strong as in figure 11a.

To investigate which of the OEC information benefit classification the most, the experiment was repeated with subsets of the binary OEC variables introduced in Eq. (20) and Eq. (21). Table 3 shows error rates starting from the case $m$ ?-v?- $\alpha$ ? shown in figure $11 \mathrm{~b}$ to the case $\mathrm{m} !-\mathrm{v}$ !- $\alpha$ ! shown in figure $11 \mathrm{c}$, as well as for all possible combinations of known and unknown OECs. For example, in the case of m?-v!- $\alpha$ ? only three additional features $v_{1} \ldots v_{3}$ were attached to the feature vector $\boldsymbol{x}_{\boldsymbol{i}}$ (Eq. (20)). Mean error rates and standard error estimates were determined using 5 -fold cross-validation on the undamaged datasets. The outlier rate in Baseline was set to 0.05. The Testsets $(\mathrm{T})$ show nearly constant missclassification rates of 0.19-0.20. This agrees well with the 0.82 ratio of feature-complete Baseline datasets (see figure 7). The missclassification rate for $\mathrm{m}$ ?-v?- $\alpha$ ? is 0.42 . However, it can be concluded from the Testset missclassification rate that approximately $20 \%$ of the observations in Damaged will be classified as outliers due to missing modes. Hence, the true missclassification rate on "good" feature vectors is even larger (approximately 50\%). The error rate can be improved substantially when information about all OECs is considered. The evaluation shows that the largest improvements can be achieved when the mass variability is explicitly measured. Knowledge about the velocity variability still improves the classification results considerably. Explicit provision of the AOA measurement does not significantly improve the error rate. Neither the direct MD approach nor the LR approach shows a decisive advantage over the other when information about the mass variability is introduced. For the $\mathrm{m}$ ? cases the direct MD approach shows slightly better error rates and significantly lower dispersion. This indicates a higher robustness to outliers. As expected the error rate improves when direct measurements of the OEV are included into the feature vector. Interestingly, separating the data into subsets and training multiple models may be a significantly better strategy than including OEV measurements into a single model as is evident from the figures 11a and 11c. Of course, this is only an option if the data can be divided into clearly separated states.

Aircraft wings or wind turbine blades rarely encounter unmeasured and local mass changes. Fuel is uniformly distributed inside a modern aircraft wing. The fuel level changes uniformly as well and the current fuel mass is usually well known during operation. The only unmeasured mass variability typically encountered by aircraft wings and wind turbine blades is icing. However, icing usually affects a large portion of the wing and therefore results in a more global modification of the mass matrix. Additional masses are sometimes attached below a wing in scientific or military applications. However, in this case 
Table 3: Mean missclassification rates $\bar{e}$ and standard error estimates $\hat{\sigma}_{e}$ for different extents of explicit OEC knowledge. OECs marked as ? were unknown during training and testing. OECs marked as ! were part of the feature vector or were used for regression. Direct application of the Mahalanobis test (MD), linear regression and a subsequent Mahalanobis test (LR). B stands for baseline data, $\mathrm{T}$ for a test set and $\mathrm{D}$ for samples of the damaged state.

\begin{tabular}{|c|c|c|c|c|c|c|c|c|c|c|c|c|c|c|c|c|c|}
\hline & & \multicolumn{2}{|c|}{$\mathrm{m} ?-\mathrm{v} ?-\alpha ?$} & \multicolumn{2}{|c|}{$\mathrm{m} ?-\mathrm{v} ?-\alpha !$} & \multicolumn{2}{|c|}{$\mathrm{m} ?-\mathrm{v} !-\alpha ?$} & \multicolumn{2}{|c|}{$\mathrm{m} ?-\mathrm{v} !-\alpha !$} & \multicolumn{2}{|c|}{$\mathrm{m} !-\mathrm{v} ?-\alpha ?$} & \multicolumn{2}{|c|}{$\mathrm{m!-v} ?-\alpha !$} & \multicolumn{2}{|c|}{$\mathrm{m} !-\mathrm{v} !-\alpha ?$} & \multicolumn{2}{|c|}{$\mathrm{m} !-\mathrm{v} !-\alpha !$} \\
\hline & & $\bar{e}$ & $\hat{\sigma}_{e}$ & $\bar{e}$ & $\hat{\sigma}_{e}$ & $\bar{e}$ & $\hat{\sigma}_{e}$ & $\bar{e}$ & $\hat{\sigma}_{e}$ & $\bar{e}$ & $\hat{\sigma}_{e}$ & $\bar{e}$ & $\hat{\sigma}_{e}$ & $\bar{e}$ & $\hat{\sigma}_{e}$ & $\bar{e}$ & $\hat{\sigma}_{e}$ \\
\hline \multirow{3}{*}{$\mathrm{MD}$} & B & 0.05 & 0.00 & 0.05 & 0.00 & 0.05 & 0.00 & 0.05 & 0.00 & 0.05 & 0.00 & 0.05 & 0.00 & 0.05 & 0.00 & 0.05 & 0.00 \\
\hline & $\mathrm{T}$ & 0.19 & 0.02 & 0.19 & 0.02 & 0.20 & 0.02 & 0.20 & 0.02 & 0.19 & 0.02 & 0.19 & 0.02 & 0.19 & 0.02 & 0.19 & 0.02 \\
\hline & $\mathrm{D}$ & 0.42 & 0.03 & 0.42 & 0.02 & 0.35 & 0.02 & 0.33 & 0.02 & 0.29 & 0.12 & 0.29 & 0.13 & 0.24 & 0.10 & 0.22 & 0.10 \\
\hline \multirow{3}{*}{ LR } & B & & & 0.05 & 0.00 & 0.05 & 0.00 & 0.05 & 0.00 & 0.05 & 0.00 & 0.05 & 0.00 & 0.05 & 0.00 & 0.05 & 0.00 \\
\hline & $\mathrm{T}$ & & & 0.19 & 0.02 & 0.20 & 0.02 & 0.20 & 0.02 & 0.20 & 0.02 & 0.19 & 0.02 & 0.19 & 0.02 & 0.19 & 0.02 \\
\hline & $\mathrm{D}$ & & & 0.44 & 0.06 & 0.39 & 0.10 & 0.38 & 0.13 & 0.28 & 0.14 & 0.29 & 0.15 & 0.22 & 0.11 & 0.20 & 0.10 \\
\hline
\end{tabular}

the data can be split into well separated states in accordance with the discussion above. Hence, joint unmeasured mass and wind variability may not be a practical problem for aircraft wings or wind turbine blades. Of course, for other applications like bridges subjected to heavy-duty traffic it could very well be.

Without further processing the false alarm rate of the proposed methodology is quite high if a consistency thresholds is chosen that is significantly lower than 100\%. Another outlier detection step could be used before the actual damage detection to identify feature vectors that contain a missing mode. However, there is always the danger of removing legitimate (damage-induced) anomalies in the data during preprocessing. Instead of an additional outlier detection step, one can take advantage of the fact that once damage occurs it does not go away. Hence, multiple successive outliers are a sure sign of an anomaly. How many consecutive outliers are required to trigger an alarm can be estimated from the false positive rate estimate obtained from the cross-validation of the training data. Assuming random occurrence of outliers and a false alarm rate of 0.2 one can be $99 \%$ confident of an anomaly after three consecutive outliers. Since this procedure effectively allows one to control the (final) false alarm rate, the trade off between the proportion of feature-complete datasets and the number of modes in the feature vector can be shifted towards the latter. More damage-sensitive features generally will improve damage detection as well as allow for better separation between damage and OEV [17]. However, there is a limit to the reduction of the consistency threshold. At some point modes may be included into the feature vector that are not excited under certain OEV at all. Hence, the assumption of random false alarm occurrences is violated and the procedure will identify this OEC as an anomaly.

The sensor locations can also influence the damage-sensitivity of an AOMA-based SHM methodology. The proposed feature vector (Eq. (16)) only includes global modal properties (natural frequencies and damping ratios). Thus, the damage-sensitivity of the feature vector is independent of the sensor locations. Since it can be shown that a given natural frequency is only unaffected by damage when the damage location coincides with a modal node of the correspondent mode shape [31], the inclusion of more natural frequencies should in general improve the damage-sensitivity of the approach. Hence, with a feature vector consisting only of global modal properties the most beneficial sensor distribution would be one that maximises the number of consistently detectable modes. However, it can also be shown that mode shape changes induced by damage are most pronounced in the vicinity of the damage location $[4,32]$. Thus, if mode shape related quantities are included in the feature vector (not further investigated in this work) it may be beneficial to place some sensors in the vicinity of potentially critical damage locations, irrespective of whether these locations are optimal from an identification point of view.

Mahalanobis distance-based anomaly detection works best for multivariate normal baseline data and may fail if this assumption is strongly violated. The experimental data used in this study are markedly non-normal (see figure 10). Still, the investigated damage case produced feature vector changes that are outside of the ellipsoid that covers $95 \%$ of the baseline data under varying wind excitation. Obviously, these results cannot be generalized to all types of damage or OEV. If a damage produces changes that fall inside the normal operation domain boundary this damage scenario is simply not detectable [17]. 
If the domain boundary is highly non-linear a more flexible domain description technique, like Support Vector Domain Description (SVDD) [33] or an Auto-Associative Neural Network (AANN) [19], may be more appropriate. However, more flexible models usually have to be parameterized using cross-validation on target and outlier data and sufficient representative samples of the latter are seldomly available in damage detection applications [33]. In general, detectability has to be proven on a case-by-case basis for each individual combinations of damage type, monitored structure and encountered OECs. Continuous monitoring for baseline data accumulation under $\mathrm{OEV}$ is comparatively simple or at least feasible for many engineering structures. This is not the case for experimentally generated outlier samples. The use of modal parameters as damage-sensitive features opens the possibility to create outlier samples from numerical models (except for damping ratios, which cannot be predicted with numerical modal analysis). However, this presupposes the availability of an accurate numerical model, knowledge about the most critical and frequent damage scenarios, valid linear approximations of the damage cases and no interdependency between damage- and OEC-induced changes to the feature vector. These are very high demands. However, if they are fulfilled, this information can furthermore be processed to investigate higher levels of SHM like type classification, severity estimation and even prognosis [16].

\section{Conclusions}

Natural frequencies and damping ratios that were automatically extracted with operational modal analysis were used to detect a small scale impact damage on a composite cantilever that was subjected to wind and mass-induced operational and environmental variability in a wind tunnel.

An automated baseline or training set preparation procedure was described and successfully tested with the experimental data from the wind tunnel. The proposed methodology is most helpful in cases where the structure under consideration has modes that are not consistently detectable under all operational and environmental conditions. The amount of user interaction was reduced to the selection of a single threshold: the desired consistency of the least well detected mode that is accepted as feature for subsequent damage detection.

It was shown that the operational variability induced significantly stronger modal parameter changes than the investigated damage. Still, the damage could be detected under varying wind velocity and angle of attack with great reliability and without information about the operational conditions during training or testing. Damage detection under joint mass and wind variability was less successful and the damage was not reliably detectable under the majority of investigated operational conditions. The damage detection performance under this conditions could significantly be improved using data normalization techniques. Finally, a simple procedure to reduce the final false alarm rate and simultaneously increase the damage sensitivity of the anomaly detection system is discussed.

\section{References}

[1] H. Sohn, C. R. Farrar, F. M. Hemez, D. D. Shunk, D. W. Stinemates, B. R. Nadler, J. J. Czarnecki, A Review of Structural Health Monitoring Literature: 1996-2001, Los Alamos and N.M, 2004.

[2] D. Montalvao, A Review of Vibration-based Structural Health Monitoring with Special Emphasis on Composite Materials, The Shock and Vibration Digest 38 (4) (2006) 295-324.

[3] J.-J. Sinou, Mechanical Vibrations: Measurement, Effects and Control, chap. A Review of Damage Detection and Health Monitoring of Mechanical Systems from Changes in the Measurement of Linear and Non-linear Vibrations, Nova Science Publishers, 643-702, 2009.

[4] F. Wei, Q. Pizhong, Vibration-based Damage Identification Methods: A Review and Comparative Study, Structural Health Monitoring 10 (1) (2011) 83-111.

[5] F. Magalhães, Á. Cunha, E. Caetano, Online Automatic Identification of the Modal Parameters of a Long Span Arch Bridge, Mechanical Systems and Signal Processing 23 (2) (2009) 316-329. 
[6] E. Reynders, J. Houbrechts, G. de Roeck, Fully Automated (Operational) Modal Analysis, Mechanical Systems and Signal Processing 29 (2012) 228-250.

[7] C. Devriendt, F. Magalhaes, W. Weijtjens, G. de Sitter, A. Cunha, P. Guillaume, Structural Health Monitoring of Offshore Wind Turbines using Automated Operational Modal Analysis, Structural Health Monitoring 13 (6) (2014) 644-659.

[8] C. Rainieri, G. Fabbrocino, Development and Validation of an Automated Operational Modal Analysis Algorithm for Vibration-based Monitoring and Tensile Load Estimation, Mechanical Systems and Signal Processing 60-61 (2015) 512-534.

[9] B. Peeters, G. de Roeck, One-year Monitoring of the Z24-Bridge: Environmental Effects Versus Damage Events, Earthquake Engineering \& Structural Dynamics 30 (2) (2001) 149-171.

[10] C. Rainieri, G. Fabbrocino, E. Cosenza, Near Real-time Tracking of Dynamic Properties for Standalone Structural Health Monitoring Systems, Mechanical Systems and Signal Processing 25 (8) (2011) 3010-3026.

[11] F. Magalhães, A. Cunha, E. Caetano, Vibration based Structural Health Monitoring of an Arch Bridge: From Automated OMA to Damage Detection, Mechanical Systems and Signal Processing 28 (2012) 212-228.

[12] W.-H. Hu, E. Caetano, Á. Cunha, Structural Health Monitoring of a Stress-ribbon Footbridge, Engineering Structures 57 (2013) 578-593.

[13] A. Deraemaeker, E. Reynders, G. de Roeck, J. Kullaa, Vibration-based Structural Health Monitoring using Output-only Measurements under Changing Environment, Mechanical Systems and Signal Processing 22 (1) (2008) 34-56.

[14] J. Schwochow, G. Jelicic, Automatic Operational Modal Analysis for Aeroelastic Applications, in: Proceedings of the 6th International Operational Modal Analysis Conference, Gijón, Spain, 2015.

[15] E. Reynders, G. Wursten, G. de Roeck, Output-only Structural Health Monitoring in Changing Environmental Conditions by Means of Nonlinear System Identification, Structural Health Monitoring doi: $10.1177 / 1475921713502836$.

[16] C. R. Farrar, K. Worden, Structural Health Monitoring: A Machine Learning Perspective, John Wiley \& Sons, Ltd, Chichester, United Kingdom, 2012.

[17] H. Sohn, Effects of Environmental and Operational Variability on Structural Health Monitoring, Philosophical Transactions of the Royal Society A: Mathematical, Physical and Engineering Sciences 365 (1851) (2007) 539-560.

[18] V. Hodge, J. Austin, A Survey of Outlier Detection Methodologies, Artificial Intelligence Review 22 (2) (2004) 85-126.

[19] E. Figueiredo, G. Park, C. R. Farrar, K. Worden, J. Figueiras, Machine Learning Algorithms for Damage Detection under Operational and Environmental Variability, Structural Health Monitoring 10 (6) (2011) 559-572.

[20] J. R. Wright, J. E. Cooper, Introduction to Aircraft Aeroelasticity and Loads, John Wiley \& Sons, Ltd, Chichester, United Kingdom, 1st edn., 2007.

[21] D. Tang, E. H. Dowell, Experimental and Theoretical Study on Aeroelastic Response of HighAspect-Ratio Wings, AIAA Journal 39 (8) (2001) 1430-1441.

[22] E. Neu, F. Janser, A. A. Khatibi, A. C. Orifici, Operational Modal Analysis of a Cantilever in a Wind Tunnel using Optical Fiber Bragg Grating Sensors, in: Proceedings of the 6th International Operational Modal Analysis Conference, Gijón, Spain, 2015.

[23] E. Neu, F. Janser, A. A. Khatibi, C. Braun, A. C. Orifici, Operational Modal Analysis of a Wing Excited by Transonic Flow, Aerospace Science and Technology 49 (2016) 73-79. 
[24] G. E. P. Box, D. R. Cox, An Analysis of Transformations, Journal of the Royal Statistical Society. Series B (Methodological) 26 (2) (1964) p 211-252.

[25] J. F. Hair, W. C. Black, B. J. Babin, R. E. Anderson, Multivariate Data Analysis, Pearson Education Limited, Harlow, United Kingdom, 7th edn., 2014.

[26] T. Hastie, R. Tibshirani, J. H. Friedman, The Elements of Statistical Learning: Data Mining, Inference, and Prediction, Springer, New York, USA, 2nd edn., 2009.

[27] J. M. Cimbala, Outliers: Lecture on Instrumentation, Measurements, and Statistics, URL http://www.mne.psu.edu/cimbala/me345/, accessed: 12 Juli, 2016, 2011.

[28] A. Deraemaeker, K. Worden, M. Belhaq, On the Use of the Mahalanobis Squared-distance to Filter out Environmental Effects in Structural Health Monitoring, in: Proceedings of the 2nd International Conference on Structural Nonlinear Dynamics and Diagnosis, Agadir, Morocco, 2014.

[29] E. J. Cross, G. Manson, K. Worden, S. G. Pierce, Features for Damage Detection with Insensitivity to Environmental and Operational Variations, Proceedings of the Royal Society A: Mathematical, Physical and Engineering Sciences 468 (2148) (2012) 4098-4122.

[30] P. Moser, B. Moaveni, Environmental Effects on the Identified Natural Frequencies of the Dowling Hall Footbridge, Mechanical Systems and Signal Processing 25 (7) (2011) 2336-2357.

[31] G. Hearn, R. B. Testa, Modal Analysis for Damage Detection in Structures, Journal of Structural Engineering 117 (10) (1991) 3042-3063.

[32] K. Roy, S. Ray-Chaudhuri, Fundamental Mode Shape and its Derivatives in Structural Damage Localization, Journal of Sound and Vibration 332 (21) (2013) 5584-5593.

[33] D. M. Tax, R. P. Duin, Support Vector Domain Description, Pattern Recognition Letters 20 (11-13) (1999) 1191-1199. 